Ina E. Bieber und Evelyn Bytzek

\title{
Herausforderungen und Perspektiven der empirischen Wahlforschung in Deutschland am Beispiel der German Longitudinal Election Study (GLES)
}

\begin{abstract}
Empirical electoral research in Germany has reached a high level of quality both with regard to theoretical approaches and methodological issues. Moreover, it is strongly linked to other disciplines in political science such as comparative political studies or political communication and also highly integrated in international electoral research. However, a changing context of voting and internal deficits are imposing challenges on electoral research in Germany which should be met in the next years and decades. Still, these challenges can also be regarded as perspectives for future electoral research. This paper thus describes the current standing of empirical electoral research in Germany and gives a detailed account of the challenges resulting from this. Further, using the example of the German Longitudinal Election Study (GLES) we show how these challenges can be and are actually met.
\end{abstract}

\section{Einleitung}

In einem repräsentativen politischen System wie dem deutschen stellen Wahlen die wichtigste Verbindung zwischen Bürger/innen und Politik dar: So sind Wahlen - zumindest auf nationaler Ebene - einerseits die einzige direkte Möglichkeit der Einflussnahme von Bürgern auf die Politik. Durch Wahlen wird folglich der Grundsatz der Volkssouveränität in demokratischen Systemen in die Tat umgesetzt. Allein aus demokratietheoretischer Sicht sind Wahlen somit lohnenswerte Untersuchungsobjekte. Andererseits werden Wahlen im Vergleich zu anderen Formen politischer Partizipation trotz sinkender Wahlbeteiligungsraten noch immer von einem erheblichen Bevölkerungsanteil genutzt, um den eigenen politischen Willen auszudrücken. Wahlen sind daher nicht nur theoretisch, sondern auch praktisch die wichtigste Verbindung zwischen Bürger/innen und Politik in Deutschland. Im Vordergrund der empirischen Untersuchung von Wahlen stehen folglich die individuellen Entscheidungen der Wähler/innen und dabei die Frage, wie diese zustande kommen. Dies ist das Forschungsfeld der empirischen Wahlforschung (Falter/Schoen 2005).

Trotz großer Erfolge, wie der Etablierung im Kanon der Politikwissenschaft oder methodischen Fortschritten, steht die empirische Wahlforschung in Deutsch- 
land jedoch vor etlichen Herausforderungen, die es in den nächsten Jahren zu meistern gilt. Diese liegen zum einen in den äußeren Bedingungen von Wahlforschung begründet, die in den letzten Jahrzehnten einen rapiden Wandel zeitigten, wie beispielsweise der Individualisierung der Gesellschaft oder Veränderungen der Medienlandschaft und damit verbunden auch der Wahlkampfführung (Beck 1983; Lachat 2007; Kohler 2005; Müller/Klein 2012; Schoen 2005a). Zum anderen muss sich auch die Wahlforschung einige Versäumnisse vorwerfen lassen, wie beispielsweise das Außerachtlassen des politischen Kontexts oder mikropsychologischer Erklärungsmechanismen für Einstellungsbildung und politisches Handeln (Gabriel/Keil 2005), die es aufzuholen gilt. Diese Herausforderungen stellen jedoch auch Perspektiven für die zukünftige Forschung dar und werden somit angenommen, am sichtbarsten durch die German Longitudinal Election Study (GLES), die diesen durch ein komplexes und elaboriertes Studiendesign begegnet (Schmitt-Beck et al. 2010a).

Der vorliegende Beitrag beschreibt daher die aktuellen Herausforderungen der deutschen Wahlforschung. Hierfür wird zunächst ein Überblick über den Stand der Wahlforschung in Deutschland anhand ihrer wichtigsten Theorien und Methoden gegeben. Darauf aufbauend werden die aktuellen Herausforderungen beschrieben und schließlich der Umgang mit diesen durch die GLES aufgezeigt. Eine Zusammenfassung mit Fazit schließst den Beitrag ab.

\section{Stand der Wahlforschung in Deutschland}

Streng genommen kann man erst für die Zeit nach dem 2. Weltkrieg von der Herausbildung einer empirischen Wahlforschung in Deutschland sprechen, da erst ab diesem Zeitpunkt national repräsentative Umfragen durchgeführt wurden. So stellt die Kölner Wahlstudie von 1961 die erste akademisch verankerte Untersuchung dieser Art mit Fokus auf Wahlverhalten dar (Kaase 2000; SchmittBeck et al. 2010a). Hierdurch wurde eine dezidiert mikrosoziologische Perspektive eingenommen, in der das Individuum mit seinen Einstellungen und Handlungen im Mittelpunkt steht. Dies unterscheidet sich stark von den zuvor praktizierten empirischen Zugängen, die aufgrund des Mangels an Daten auf der Individualebene Aggregatdaten auf regionaler Ebene heranzogen (Roth 2008). Auch wenn die Kölner Wahlstudie von 1961 methodisch und theoretisch sehr breit angelegt und somit nicht nur auf Umfragen beschränkt war, hat sich die national repräsentative Umfrage schnell zur Standardmethode der Datenerhebung in der empirischen Wahlforschung entwickelt, so dass zu jeder Bundestagswahl seit 1961 eine solche Datenquelle vorliegt. ${ }^{1}$ Neben dem Standardinstrument der Vor- und Nachwahlquerschnittsbefragung kamen in jüngerer Zeit auch Längsschnitt-Untersuchungen, wie beispielsweise mehrere Wahljahre umspannende Panelbefragungen oder den Wahlkampf abbildende Rolling-Cross-SectionUmfragen, hinzu (Schmitt-Beck 2012a). Wie schon an der Verfeinerung der Umfragedesigns erkennbar ist, konnten insbesondere in Hinblick auf die Stichprobenziehung und die Nutzbarmachung neuer Umfragemodi beachtliche methodische

\footnotetext{
${ }^{1}$ Für einen Überblick zur Entwicklung der Wahlforschung in Deutschland siehe Kaase 2000.
} 
Fortschritte verzeichnet werden. Nachdem Wahlumfragen zunächst persönlichmündlich (,face-to-face') durchgeführt wurden, begann in den 1980er Jahren der Siegeszug der telefonischen Umfragen aufgrund ihres elaborierten Stichprobendesigns bei gleichzeitig kostengünstiger Durchführung (Schroth 2003). Mit Online-Umfragen steht der empirischen Wahlforschung in der jüngsten Zeit eine weitere Möglichkeit der Datenerhebung zur Verfügung, die noch kostengünstiger als Telefonumfragen ist. Ein weiterer Vorteil von Online-Umfragen ist, dass in einer kurzen Erhebungszeit viele Interviews durchgeführt werden können, die darüber hinaus die Möglichkeit bieten, grafische Elemente bis hin zu Video- und Audiodateien in das Interview einzubauen. Aufgrund der bislang nicht flächenmäßigen Internetabdeckung sind Online-Umfragen jedoch mit starken Repräsentativitätsproblemen behaftet, da viele wahlberechtigte Personen aufgrund des Fehlens eines Internetanschlusses eine Auswahlwahrscheinlichkeit von Null haben (zum Nutzen von Online-Umfragen für die Wahlforschung siehe Bieber /Bytzek 2012). Dennoch gewinnt diese Form der Datenerhebung nicht nur für Bevölkerungsumfragen an Bedeutung, sondern auch für die Befragung von Kandidat/innen zur Bundestagswahl und Wahlkampfmanager/innen der Parteien, die zuvor nur durch den Versand von Fragebögen erfolgen konnte (Tenscher 2007; Zittel/Gschwend 2007). Insbesondere durch den Fokus auf Umfragen als Methode der Datenerhebung hat die Wahlforschung einen wesentlichen Beitrag zur Entwicklung der empirischen Sozialforschung geleistet (Noelle-Neumann 2000).

Trotz des Fokus auf Umfragen spielen weitere Formen der Datenerhebung eine Rolle für die empirische Wahlforschung. In erster Linie sind hier Medieninhaltsanalysen zu nennen, die den medialen Kontext einer Wahl erfassen. Doch obwohl im Rahmen des integrierten Designs der Kölner Wahlstudie von 1961 bereits eine quantitative Inhaltsanalyse von Zeitungen und Fernsehsendungen enthalten war (Scheuch 2000), wurde dies bis zur Bundestagswahl 2005 nicht weitergeführt. Medieninhaltsanalysen wurden folglich in der Regel von Medien- und Kommunikationswissenschaftler/innen durchgeführt, die an Politischer Kommunikation interessiert sind (stellvertretend hierfür zur Bundestagswahl 2005 Boomgaarden/Semetko 2007; zur Bundestagswahl 2002 Brettschneider/Rettich 2005). So werden zumeist mit diesen Medieninhaltsanalysen spezielle Fragestellungen untersucht, durch die nur wenige Medien anhand eines auf die Fragestellung zugeschnittenen Kategorienschemas erfasst werden. Hierdurch ist es schwierig, Verbindungen zwischen den größtenteils sehr speziellen Medieninhaltsanalysen und den thematisch eher breiten Wahlumfragen herzustellen und somit Wirkungen des medialen Kontexts auf Wahlentscheidungen stringent zu erfassen. Dies ist einer der Gründe dafür, dass in der jüngsten Zeit vermehrt auch experimentelle Designs verwendet werden, um die Wirkung politischer Informationen auf Wahlentscheidungen zu untersuchen (Faas/Huber 2010). Exemplarisch sollen hier die Studien zur Wirkung von TV-Duellen herausgegriffen werden, bei denen Probanden darum gebeten werden, bei Betrachtung des TV-Duells unter Laborbedingungen, d.h. in Räumen unterschiedlicher Universitäten, mit Hilfe eines Drehreglers ständig ihre Bewertung der beiden Kandidaten kundzutun (Maier/Faas 2005; Maier/Maier 2007; Maier et al. 2013). Der empirischen Wahlforschung 
stehen somit qualitativ hochwertige und innovative Möglichkeiten der Datenerhebung zur Verfügung, die ebenso wie die Verwendung elaborierter statistischer Methoden zur Auswertung dieser Daten zum Aufbau ihres Rufs als technisch versiertem und präzisem Wissenschaftszweig beigetragen haben. Selbstverständlich sind diese Datenerhebungsmöglichkeiten auch mit erheblichen Problemen belastet, die in Teil 3 dieses Beitrags beschrieben werden.

In Hinblick auf gängige Theorien hat sich die empirische Wahlforschung vor allen Dingen bei den US-Kollegen bedient, so dass drei theoretische Ansätze vorherrschend sind: das mikrosoziologische, das sozialpsychologische und das ökonomische Modell der Wahlentscheidung (Falter/Schoen 2005; Pappi/Shikano 2007; für einen knappen Überblick Schmitt-Beck 2012a). Das mikrosoziologische Modell geht auf Lazarsfeld et al. (1968) zurück und erklärt die Wahlentscheidung durch die Zugehörigkeit der Wähler/innen zu einer sozialen Gruppe wie beispielsweise die der Katholik/innen oder Arbeiter/innen. Die Einbindung des Individuums in diese einstellungshomogenen Gruppen übt somit den sozialen Druck aus, durch den sich aus sozialen Merkmalen politisches Verhalten ergibt (Schmitt-Beck 2012a). Um die Bindung der sozialen Gruppen an Parteien zu erklären, wurde dieses mikrosoziologische Modell von Lipset und Rokkan (1967) um eine makrosoziologische Perspektive ergänzt, die soziale Gruppen und Parteien über sogenannte gesellschaftliche Konfliktlinien, auf denen sowohl Gruppen als auch Parteien Position beziehen, miteinander verbindet. Diese Konfliktlinien umfassen den Konflikt zwischen Arbeit und Kapital, zwischen Stadt (sekundärer Sektor) und Land (primärer Sektor), Zentrum und Peripherie sowie Kirche und säkularem Staat (Pappi/Shikano 2007; Schoen 2005b).

Die Zugehörigkeit zu sozialen Gruppen spielt auch im sozialpsychologischen Modell von Campbell et al. (1960) eine Rolle, wirkt jedoch nicht direkt auf das Wahlverhalten, sondern vermittelt über politische Einstellungen. Grundlage des Modells ist folglich ein Kausalitätstrichter, an dessen schmalen Ende die individuelle Wahlentscheidung steht. Zeitlich nah daran verortet sind politische Einstellungen, auf die wiederum stabilere, jedoch zeitlich weiter entfernte soziale Merkmale wirken. Da diese Einstellungen der Wahlentscheidung direkt vorgelagert sind, steht folglich deren Betrachtung im Vordergrund des Modells. Sie werden in langfristige (Parteiidentifikation) und kurzfristige (Sachfragen- und Kandidatenorientierung) Einflussfaktoren unterschieden, wobei die langfristig stabile Parteiidentifikation laut des Originalmodells kurzfristige Einstellungen beeinflusst (Schoen/Weins 2005). Insbesondere der Stellenwert der Parteiidentifikation, die zunächst als langfristig stabile, affektive Bindung an eine Partei konzipiert wurde, ist jedoch umstritten, so dass nun Ansätze überwiegen, die auch die Parteiidentifikation nicht als unveränderlich betrachten, sondern als Resultat der Erfahrungen mit den Parteien (Fiorina 1981).

Das ökonomische bzw. Rational-Choice-Modell schließlich geht auf Downs (1957) zurück und verfolgt einen gänzlich anderen Ansatz: Es verortet Wähler/innen sowie Parteien in einem politischen Sachfragenraum, in dem die Wähler/innen als Nachfrager von Politik auftreten und diese mit ihrer Stimme ,kaufen', während die Parteien Politik in Form von Wahlprogrammen anbieten. Dem Kriterium der Nutzenmaximierung folgend wird die Partei gewählt, die dem/der 
Wähler/in in diesem Raum am nächsten steht. Die Wähler/innen vergleichen folglich die Wahlprogramme der Parteien und bewerten diese in Hinblick auf ihre eigenen politischen Präferenzen. Auch die Parteien verhalten sich nutzenmaximierend und positionieren sich aufgrund des Ziels der Stimmenmaximierung so, dass sie ihre Distanz zu möglichst vielen Wähler/innen reduzieren (Distanzwahl). Dieser Ansatz fokussiert folglich auf das Kerngeschäft der Politik, den politischen Inhalten, blendet dabei aber einerseits aus, wie die Präferenzen der Wähler/innen entstehen. Andererseits nimmt das Modell an, dass die Wähler/innen vollständige Informationen über die Programme der Parteien besitzen, was reichlich unrealistisch ist. Daher wurde auch dieses Modell modifiziert, beispielsweise in Hinblick auf weniger anspruchsvolle Rationalitätskonzepte oder der Postulierung einer Richtungswahl in Konkurrenz zur Distanzwahl, bei der die Wähler/innen die Partei wählen, die ihre politische Grundrichtung am extremsten vertritt (Arzheimer/Schmitt 2005; Pappi/Shikano 2007; Schmitt-Beck 2012a).

Diese drei grundlegenden Modelle wurden von der deutschen empirischen Wahlforschung in Hinblick auf ihre Tauglichkeit zur Abbildung bundesdeutscher Verhältnisse überprüft sowie Konzepte und Messungen gegebenenfalls angepasst (für das soziologische Modell beispielsweise Pappi 1973; 1977; für das sozialpsychologische Modell beispielsweise Falter et al. 2000; Rattinger 1998; für das ökonomische Modell beispielsweise Pappi 1989; Thurner 1998). Neben diesen drei Ansätzen spielen weitere theoretische Modelle in der deutschen empirischen Wahlforschung eine Rolle, die den Fokus von der Mikro- auf die Makroebene lenken, wie in Untersuchungen zum Zusammenhang zwischen Wirtschaftslage und Regierungspopularität (Pappi/Shikano 2007). Ein thematischer Bereich der Wahlforschung soll hier besondere Erwähnung finden, da sich deutsche Wahlforscher/innen hier international hervorgetan haben: das Konstrukt des ,Strategischen Wählens'. Im Vordergrund steht hier, dass sich manche Wähler/innen dazu entscheiden, ihre Stimme nicht der meistpräferierten Partei zu geben, sondern der Partei, die am höchsten in ihrer Präferenzordnung steht und der sie zugleich die größten Chancen auf den Wahlsieg einräumen. Dieses Verhalten wird auf Anreize, die das Wahlsystem bietet, im Zusammenhang mit den Koalitionspräferenzen der Wähler/innen zurückgeführt (Behnke 2008; Gschwend 2007; Herrmann/Pappi 2008; Meffert/Gschwend 2010; Pappi/Thurner 2002). Ein Grund für die große internationale Sichtbarkeit dieses Forschungsgebiets dürfte daher im deutschen Wahlsystem liegen, das mit seiner Zwei-Stimmen-Konstruktion sogar mehrere Arten von strategischem Wahlverhalten ermöglicht und somit deutliche Anreize für ein solches Verhalten bietet. Von strategischem Erststimmenwählen ist die Rede, wenn im Wettbewerb um Direktmandate, die anhand eines Mehrheitswahlsystems vergeben werden, Wähler/innen ihre Stimme nicht vergeuden wollen und damit in erster Linie CDU/CSU und SPD (in Ostdeutschland zusätzlich DIE LINKE) wählen, da abgesehen von sehr wenigen Ausnahmen nur diese Parteien eine Chance auf das Direktmandat haben. Die Vergabe einer Leihstimme als strategisches Wahlverhalten bezieht sich dagegen auf den Verhältniswahlteil des deutschen Wahlsystems, bei dem eine Sperrklausel von fünf Prozent angewendet wird. Um einer kleinen Partei, die an dieser Hürde scheitern 
könnte, den Einzug in den Bundestag zu sichern, können Anhänger/innen einer großen Partei ihre Stimme der kleinen Partei leihen, sofern sie auf eine Koalition der beiden Parteien hoffen. Darüber hinaus ist es natürlich möglich, dass auch in Hinblick auf die Fünfprozenthürde die Verschwendung der Stimme an aussichtslose Parteien vermieden wird. Das deutsche Wahlsystem ist somit nicht nur ein Exportschlager, sondern hat auch der deutschen Wahlforschung zu einer internationalen Sichtbarkeit verholfen.

Sowohl in Hinblick auf die verwendeten Methoden der Datenerhebung und -auswertung als auch der den Berechnungen zugrundeliegenden theoretischen Modelle ist die deutsche empirische Wahlforschung folglich keinen Sonderweg gegangen und in die internationale Forschergemeinschaft gut eingebunden. Dies bedeutet jedoch nicht, dass die Wahlforschung in Deutschland keinen Herausforderungen gegenübersteht, denen es in den nächsten Jahren und Jahrzehnten zu begegnen gilt, um ihre hohen wissenschaftlichen Standards weiterhin halten zu können. Diese Herausforderungen werden im nächsten Abschnitt dargestellt.

\section{Aktuelle Herausforderungen für die deutsche Wahlforschung}

\subsection{Gesellschaftliche Veränderungen und Trends}

Zu den äußeren Veränderungen, die die empirische Wahlforschung in Deutschland vor Herausforderungen stellen, ist zuallererst der allgemeine gesellschaftliche Wandel zu zählen, der häufig mit dem Begriff der Individualisierung beschrieben wird (Beck 1983; Kohler 2005; Müller/Klein 2012; für kritische Betrachtungen dieser These Clark/Lipset 1991; Müller 1997; 1999). Durch Individualisierungsprozesse in der Gesellschaft wird die Bindung von Bürger/innen zu sozialen Gruppen und Parteien zunehmend aufgelöst. Hierdurch werden die Wähler/innen schlicht unberechenbarer: Während der soziale Hintergrund und die Parteiidentifikation als hauptsächliche Erklärungsfaktoren für Wahlverhalten im Rückgang begriffen sind, werden eine Vielzahl von Kurzfristfaktoren, wie die inhaltliche Distanz zu Parteien oder die Kandidatenpräferenz, wichtiger für Wahlentscheidungen (Debus 2012a). Aufgrund dessen entscheidet sich eine immer größer werdende Zahl an Wähler/innen erst kurz vor der Wahl für eine Partei (Schmitt-Beck/Partheymüller 2012), zudem nimmt die Zahl der sogenannten Wechselwähler/innen zu, die sich von Wahl zu Wahl für andere Parteien entscheiden (Schoen 2005c; Weßels 2011a). Eine weitere Konsequenz dieser Entwicklung ist, dass man nun nicht mehr von dem Wähler oder der Wählerin ausgehen kann, dessen/deren Verhalten durch ein einzelnes zugrundeliegendes Modell erklärt werden kann, sondern von einer Vielzahl an Entscheidungslogiken ausgehen muss.

Diese Entwicklung stellt die empirische Wahlforschung in doppelter Hinsicht vor Herausforderungen: Erstens hat dies Konsequenzen für die Datenerhebung. Das ,Flaggschiff' der empirischen Wahlforschung, die vor und nach der Wahl durchgeführten persönlich-mündlichen Umfragen mit einer hohen Zahl an Be- 
fragten, kann diese kurzfristigen Dynamiken nicht abbilden. Zweitens lassen sich diese Entwicklungen nur zum Teil in die bestehenden empirischen Ansätze unterbringen. Denn auch wenn das sozialpsychologische und das rationale Modell Raum für kurzfristige Faktoren in der Wahlentscheidung lassen, zeigen empirische Befunde, dass der Anteil der Wähler/innen, die sich auf Basis von Sachfragen (entweder in Hinblick auf die wahrgenommene Kompetenz der Parteien oder deren Position in Relation zur eigenen Position) oder Kandidatenorientierungen für eine Partei entscheidet, nicht konstant steigt (Pappi/Shikano 2001; Thurner et al. 2012; Wagner/Weßels 2012a). Stattdessen scheinen die jeweils bei einer Wahl spezifischen Bedingungen den Ausschlag zu geben. Doch welche dies sind, ist bislang unbekannt.

Der Trend zu zunehmender Individualisierung wurde in Deutschland durch die deutsche Wiedervereinigung 1990 verstärkt. Hierdurch wurde das Elektorat um eine Gruppe an Wähler/innen ergänzt, die in einem völlig anderen politischen System sozialisiert wurden, so dass die Gültigkeit der bestehenden theoretischen Modelle in Frage gestellt werden muss, insbesondere des soziologischen und sozialpsychologischen Modells aufgrund des Fokus auf Langfristfaktoren. Auch wenn gezeigt werden konnte, dass ostdeutsche Bürger/innen über Einstellungen verfügen, die für diese Modelle relevant sind, so sind diese meist schwächer ausgeprägt als in der westdeutschen Wählerschaft (Falter et al. 2000; Ohr/Quandt 2012).

Ein zweiter Motor der Folgen der Individualisierung liegt in der Veränderung des elektoralen Kontexts: Durch die rasche Zunahme des Medienangebots ist einerseits die Fülle an politischen Informationen, denen die Wähler/innen ausgesetzt sind, größer geworden. Andererseits ist es hierdurch paradoxerweise auch leichter geworden, diesen Informationen aus dem Weg zu gehen und dennoch eine hohe Mediennutzung aufzuweisen, beispielsweise indem Nachrichtensendungen mit geringem politischen Inhalt im Fernsehen konsumiert werden. Hierdurch und ebenso durch die abnehmenden Parteibindungen der Wähler/innen - hat sich auch die Wahlkampfführung der Parteien verändert, die nun nicht mehr in erster Linie ihr Stammklientel mobilisieren, sondern jeden erdenklichen Wählertyp ansprechen wollen (Niedermayer 2007; Schoen 2005a; Tenscher 2007; 2013).

In dieser Medien- und Parteienvielfalt den Überblick zu bewahren, ist für die Wähler/innen nicht einfach. Hilfreich kann dabei ein erst 2002 eingeführtes Wahlkampfereignis sein: das sogenannte TV-Duell. Hier werden die Spitzenkandidat/innen der beiden großen Parteien von Moderator/innen unterschiedlicher Fernsehsender in 90 Minuten zu unterschiedlichsten politischen Themen befragt. Dieses TV-Format vermag es einerseits, den Wahlkampf in komprimierter Form abzubilden und erreicht damit sehr hohe Einschaltquoten (Maier et al. 2013; Maier/Maier 2007). Dies deutet darauf hin, dass es eine Orientierungshilfe für die Wähler/innen darstellen kann. Andererseits stehen TV-Duelle unter dem Verdacht, einem weiteren, häufig monierten Trend in der Medienberichterstattung Vorschub zu leisten: der Personalisierung der Politik (Maier et al. 2013; Maier/Maier 2007). Hierunter wird u.a. ein Fokus der Medien auf die Spitzenkandidat/innen im deutschen Fall insbesondere der beiden großen Parteien verstanden, durch den sachpolitische Fragen in den Hintergrund gedrängt werden (Brettschneider 2002). Diese, möglicherweise lediglich befürchtete Entwicklung 
lässt sich beispielsweise im sozialpsychologischen Modell der Wahlentscheidung abbilden, indem sich dieser Trend in einem höheren Einfluss der Kandidatenorientierung im Vergleich zur Sachfragenorientierung niederschlagen sollte. Unklar bleibt jedoch, welche Rolle hierbei neue Wahlkampfformate wie das TV-Duell spielen, da diese nicht stringent in bestehende Modelle der Wahlentscheidung

\subsection{Innere Probleme und Defizite}

Neben diesen äußeren Veränderungen, die die deutsche empirische Wahlforschung vor Herausforderungen stellen, lassen sich auch Defizite innerhalb dieses Forschungszweigs feststellen, die zum Teil daraus resultieren, dass erst spät auf die äußeren Veränderungen reagiert wurde. In Hinblick auf die theoretische Basis lässt sich ein gewisser „Theorieeklektizismus“ (Schoen 2009a) feststellen, der darin mündet, dass ein gemeinsames Theoriegebäude der empirischen Wahlforschung bislang fehlt. So werden neu entdeckte Erklärungsfaktoren für Wahlverhalten häufig ad hoc oder nur mit geringer Rückbindung an bestehende Theorien in Modelle einbezogen. Hierdurch bleibt unklar, in welchem Verhältnis sie zu den altbekannten Erklärungsfaktoren stehen, was wiederum die empirischen Befunde angreifbar macht. Gemeinsame Anstrengungen mit dem Ziel der Erstellung eines integrierten Modells der Wahlentscheidung sind folglich dringend angebracht.

Hinsichtlich der Methoden der Datenerhebung kämpft die empirische Wahlforschung sowohl in Hinblick auf persönlich-mündliche als auch auf telefonische Umfragen mit stark gesunkenen Ausschöpfungsquoten, die den Anspruch der Repräsentativität der Daten schmälern und somit Zweifel aufwerfen, dass die mit diesen Daten berechneten Ergebnisse auf die Gesamtbevölkerung übertragbar sind (Sanders et al. 2007). So gilt in persönlich-mündlichen Umfragen zwischenzeitlich eine Ausschöpfungsquote von ca. 50\% als gutes Ergebnis, bei telefonischen Umfragen sinkt diese bisweilen auf unter 25\% (Häder 2011, Kap. 6.1; Schmitt-Beck et al. 2010b). Dies wäre unproblematisch, wenn die Ausfälle zufällig verteilt wären, was jedoch nicht der Fall ist: Bekannterweise nehmen an Umfragen in erster Linie höher gebildete Wähler/innen teil, die nur einen Bruchteil der Bevölkerung ausmachen (Bieber/Bytzek 2012), was sich beispielsweise auf das gemessene Niveau an politischem Interesse und politischem Wissen auswirken dürfte. Bei Telefonumfragen treten durch die starke Verbreitung von Mobiltelefonen und einer zunehmenden Zahl an Haushalten, die aufgrund dessen keinen Festnetzanschluss mehr besitzt, zwei weitere Probleme auf: Einerseits stellt die Stichprobenziehung eine Herausforderung dar, andererseits wird die Interviewsituation durch die Erreichbarkeit an so ziemlich jedem Ort unkontrollierbar (Hunsicker/Schroth 2007).

Als Lösung für die Probleme mit der Ausschöpfungsquote (und den dadurch massiv gestiegenen Kosten) bei persönlich-mündlichen und telefonischen Umfragen werden häufig die bereits genannten Online-Umfragen angesehen, da sie zum einen in sehr kurzer Zeit eine hohe Zahl an Interviews generieren, zum anderen sehr kostengünstig sind. Die vorrangigen Probleme mit Online-Umfragen liegen jedoch auf der Hand: Durch die Beschränkung auf Personen mit Internetzugang kann nicht die gesamte Bevölkerung erreicht werden, insbesondere ältere 
Menschen mit niedrigem Bildungsniveau und geringem Einkommen werden systematisch ausgeschlossen. Durch eine fortschreitende Internetpenetration in der deutschen Bevölkerung besitzt dieses Instrument jedoch das Potential zukünftig zu einer wichtigen Datenerhebungsmethode in der Wahlforschung zu werden und sollte daher nicht außer Acht gelassen und idealerweise neben traditionelle Umfragemodi gestellt werden, um Effekte des Erhebungsmodus besser einschätzen zu können (Bieber/Bytzek 2012).

Zwei weitere Defizite der empirischen Wahlforschung betreffen sowohl den Bereich der zugrundeliegenden Theorien als auch die genutzten Methoden der Datenerhebung: Erstens werden trotz des Fokus auf individuelle Entscheidungen selten mikropsychologische Theorien der Einstellungsbildung und der Entscheidungsfindung herangezogen und empirisch getestet (Faas et al. 2010). Einstellungen umfassen kognitive, affektive und konative Aspekte (Rosenberg/Hovland 1960). Da der konative Aspekt bereits die Handlungsintention abdeckt, sollten vor allem stärker psychologische Theorien einbezogen werden, die die kognitiven und affektiven Bestandteile von Einstellungen, und somit die Entstehung dieser, untersuchen. In Hinblick auf kognitive - also informationsbasierte Aspekte von Einstellungen - spielt die Verarbeitung von Informationen im Zusammenspiel mit bestehenden Einstellungen eine zentrale Rolle. Dies betrifft also die Frage, wie Wähler/innen mit Informationen umgehen und welche Heuristiken sie anwenden, um mit der Flut an Informationen umgehen zu können. Daneben ist jedoch auch die Untersuchung affektiver - also emotionaler Bestandteile von Einstellungen - wichtig, da Kognition der Emotion bedarf, beides also nicht getrennt voneinander betrachtet werden kann, auch wenn die Wahlforschung diese Sichtweise gerne außer Acht lässt (Steenbergen 2010).

Das zweite Defizit in Hinblick auf die Theoriebasis liegt darin, dass auch der gesellschaftliche und politische Kontext, in dem Wahlentscheidungen getroffen werden, selten eine Rolle in der Erklärung von Wahlentscheidungen spielt (Gabriel/Keil 2005). Wie das oben genannte Beispiel des strategischen Wählens zeigt, kann die institutionelle Ausgestaltung des politischen Systems Einfluss auf Wahlverhalten nehmen. Neben diesen wenig veränderlichen Kontextbedingungen weisen zudem etliche Befunde darauf hin, dass jede Wahl anders ist, also andere Kandidat/innen, politische Programme, Kommunikationsbemühungen seitens der Parteien und auch Ereignisse den gesellschaftspolitischen Kontext einer Wahl formen und damit auch Einfluss auf das Wahlverhalten haben können (SchmittBeck 2012a). Daher gilt es zu identifizieren, welche Faktoren hierbei wichtig sind und diese stringent in ein Modell der Wahlentscheidung zu integrieren.

Sowohl die Beachtung mikropsychologischer Theorien als auch des gesellschaftspolitischen Kontexts stellt die notwendige Basis dar, um das komplexe Gebilde ,Wahlentscheidung“ konsequent auf der Mikroebene unter Einbeziehung der Makroebene, also des Kontexts, untersuchen und damit die Entscheidungssituation, in der sich die Wähler/innen befinden, adäquat abbilden zu können. Die Integration beider Perspektiven hat insofern einen Einfluss auf die Datenerhebung als dass hierdurch Umfragen einerseits durch experimentelle Designs ergänzt werden müssen, um individuelle Mechanismen der Entscheidungsfindung und Einstellungsbildung auf Basis politischer Informationen untersuchen zu kön- 
nen. Andererseits muss der gesellschaftliche und politische Kontext empirisch erfasst werden, in Hinblick auf die Informationssituation beispielsweise durch Inhaltsanalysen, die sich mit Umfragedaten in Verbindung bringen lassen.

Die empirische Wahlforschung in Deutschland steht damit zusammenfassend vor den äußeren Herausforderungen einer zunehmend individualisierten Gesellschaft sowie einer wachsenden Mediatisierung und auch Personalisierung von Politik. Diese äußeren Veränderungen führen dazu, dass es mehr als je zuvor notwendig ist, den Kontext von Wahlen zu erfassen sowie theoretische Defizite, wie das Fehlen eines integrierten Ansatzes oder das Außerachtlassen psychologischer Erklärungen zu Informationsverarbeitung und Entscheidungsfindung, zu beheben. Dies hat direkte Auswirkungen auf die Methoden der Datenerhebung, da solche Untersuchungen auf Basis des Standardinstruments, der repräsentativen Umfrage, nicht möglich sind. Darüber hinaus ist die empirische Wahlforschung auch in Hinblick auf die Datenerhebung gefordert, den durch sinkende Ausschöpfungsquoten bedrohten hohen Standard der Datenqualität aufrechtzuerhalten. Wie mit diesen Herausforderungen aktuell umgegangen wird, zeigt der nachfolgende Teil anhand der Antworten der German Longitudinal Election Study (GLES) auf die aufgeworfenen Fragen.

\section{Von den Herausforderungen zu den Perspektiven: Die German Longitudinal Election Study}

Die GLES ist das umfangreichste Projekt der deutschen Wahlforschung zur Erfassung und Erklärung des Wahlverhaltens und sie ist auch international eine der breitest angelegten Wahlstudien (Eder et al. 2010; Schmitt-Beck et al. 2010a). Sie wurde 2007 von der wissenschaftlichen Gemeinschaft unter der Leitung der Primärforscher/innen Hans Rattinger, Sigrid Roßteutscher, Rüdiger Schmitt-Beck und Bernhard Weßels ins Leben gerufen, indem Fördergelder zur Untersuchung der Bundestagswahlen 2009, 2013 und 2017 bei der Deutschen Forschungsgemeinschaft (DFG) beantragt und genehmigt wurden. Das Leibniz-Institut für Sozialwissenschaften (GESIS) unterstützt unter Leitung von Christof Wolf insbesondere die Datenaufbereitung und -distribution (GLES 2013a; Schmitt-Beck et al. 2010a). Bevor nun aufgezeigt wird, wie die GLES auf die einzelnen Herausforderungen, Probleme und Defizite der Wahlforschung reagiert, soll zunächst ein kurzer Überblick über das grundlegende Studiendesign gegeben werden.

\subsection{GLES im Überblick}

Wie nachfolgende Abbildung 1 zeigt, umfasst die GLES drei Projektphasen, die sich jeweils auf eine Bundestagswahl beziehen. Mittels insgesamt neun Komponenten werden die lang- und kurzfristigen Einflussfaktoren auf den Wahlentscheidungsprozess der Bürger/innen unter Berücksichtigung des Medieneinflusses und der Wahlkampfführung seitens der Parteien und Kandidat/innen erfasst. Die Komponenten unterscheiden sich hinsichtlich der Datenerhebungsinstrumente, der Auswahlverfahren und der thematischen Schwerpunktsetzung. Nachdem die 
Bundestagswahl 2009 erstmalig in dieser umfangreichen Weise erfasst wurde, ist eine weitere entsprechende Datenerhebung bei der Wahl 2013 erfolgt und soll auch für die Bundestagswahl 2017 umgesetzt werden. Die GLES zeichnet sich außerdem dadurch aus, dass die Daten sehr zeitnah und transparent über GESIS an die wissenschaftliche Gemeinschaft distribuiert werden und somit in sehr kurzer Zeit ein immenses öffentliches Gut produziert wird. Nach 2017 soll das Projekt in institutionalisierter Weise als German Election Study (GES) fortgeführt werden (Eder et al. 2010; Schmitt-Beck et al. 2010a).

Neben dem bereits vorgestellten ,Flaggschiff' der Wahlforschung - eines bundesweiten bevölkerungsrepräsentativen Vor- und Nachwahlquerschnitts (Komponente 1) - und dem Standardinstrument der Telefonbefragung in Form einer Rolling-Cross-Section (Komponente 2) beheimatet die GLES auch ein auf jeweils drei Bundestagswahlen angelegtes Langfrist-Panel mit Vor- und Nachwahlerhebung (Komponente 7). Um den Einfluss der Medien auf die Wahlentscheidung abschätzen zu können, wurden eine Wahlkampf-Medieninhaltsanalyse (Komponente 4) und eine computergestützte Langfrist-Medienagenda-Analyse (Komponente 9) implementiert. Ferner erfassen zwei Online-Komponenten einerseits langfristige Entwicklungen (Langfrist-Online-Tracking, Komponente 8) und andererseits kurzfristige Prozesse im Wahlkampf (Wahlkampf-Panel, Komponente 3). Der spezifischen Analyse der Kandidat/innen dienen die TV-Duell-Analyse (Komponente 5) und die Kandidatenstudie (Komponente 6).

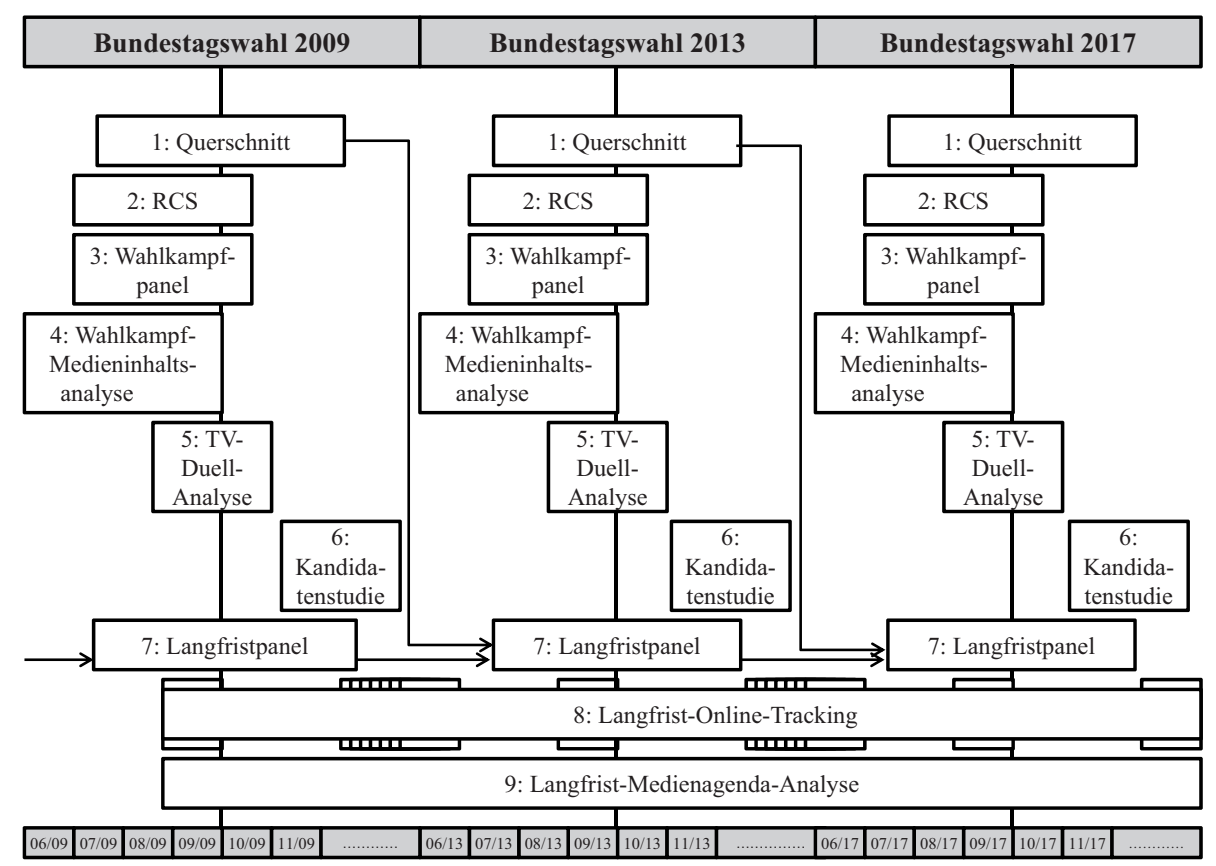

Abbildung 1: Überblick der GLES (GLES 2013b, modifiziert und erweitert) 
Besonders an diesem Studiendesign ist nicht nur die Vielfalt hinsichtlich Methoden und Inhalten, sondern auch der langfristige Charakter zahlreicher Komponenten auf Individual- und Aggregatdatenebene, die Existenz eines gemeinsamen Kernfragebogens, die Fusionsfähigkeit der einzelnen Komponenten untereinander und der Anschluss einiger Komponenten an internationale Wahlstudien: So ist das Online-Tracking beispielsweise auf die langfristige Medieninhaltsanalyse gleichermaßen abgestimmt wie die telefonische Befragung auf die WahlkampfMedieninhaltsanalyse. Auch der Vorwahl-Querschnitt steht in Verbindung zur Kandidatenstudie, welcher gleichzeitig an den Comparative Candidates Survey (CCS) angedockt ist. Der Nachwahl-Querschnitt wiederum beheimatet Fragen aus der international vergleichenden Comparative Study of Electoral Systems (CSES). Doch wie geben die einzelnen Komponenten der GLES nun spezifische Antworten auf die beschriebenen Herausforderungen und Probleme der empirischen Wahlforschung?

\subsection{Herausforderung I: Individualisierungsprozesse}

Wie zuvor dargestellt ist auch die deutsche Wahlforschung mit Individualisierungsprozessen konfrontiert. Auf diese wohl größte Herausforderung reagiert die GLES in verschiedenen Komponenten: Zunächst baut die QuerschnittKomponente (Komponente 1, ZA5302) auf den repräsentativen Bevölkerungsumfragen seit 1994 auf und ermöglicht durch seither vergleichbare Frageformulierungen die Analyse langfristiger Änderungen (Rattinger et al. 2012a). So ist es möglich auf Aggregatdatenebene zu untersuchen, ob langfristige Faktoren bei der Wahlentscheidung weniger wichtig und somit infolge der Individualisierung tatsächlich kurzfristige Faktoren einflussreicher werden (vgl. hierzu Dalton 2012; Debus 2012; Rattinger et al. 2011a; Roßteutscher 2012; Thurner et al. 2012; Wagner/Weßels 2012a).

Für die Analyse langfristiger Änderungen auf Individualebene bieten sich die Daten des Langfrist-Panels (Komponente 7, ZA5320, ZA5321) an, bei welchem jeweils anlässlich dreier aufeinanderfolgender Bundestagswahlen zu jeder Wahl dieselben Personen erneut befragt werden. Dadurch kann beispielsweise herausgefunden werden, ob über die Zeit hinweg bei ein und demselben Individuum kurzfristige Faktoren immer wichtiger werden. Das Langfrist-Panel führt 2002 und 2005 gestartete Panelbefragungen fort (vgl. Rattinger et al. 2012b; 2012c). Grundlage der 2009 gestarteten Panelwelle bilden die Personen der Querschnittbefragung aus Komponente 1 (Schmitt-Beck et al. 2010a), wodurch Synergieeffekte zwischen den einzelnen Komponenten erzeugt werden.

Obwohl die Individualisierungsthese häufig damit beschrieben wird, dass die Einflüsse von bestimmten Gruppenmerkmalen auf die Wahlentscheidung zugunsten einer spezifischen Partei immer unbedeutender werden (Schoen/Weins 2005), wird auch argumentiert, dass spezifische Gruppen, wie z.B. in Gewerkschaften organisierte oder religiös gebundene Personen, in jüngster Zeit auf der Aggregatebenen zwar an Einfluss einbüßen, jedoch für die individuelle Wahlentscheidung derjenigen, die diesen Gruppen verbunden sind, weiterhin durchaus Erklärungskraft bieten (vgl. hierzu bspw. Elff 2007; Elff/Roßteutscher 2011; Roßteutscher 
2007; Weßels 2011b). Um auch kleine Wähler/innengruppen und derartige Thesen zu untersuchen, werden die Interviews mit einer verhältnismäßig großen Fallzahl von ca. 4200 Vorwahl- und Nachwahlbefragten in der Querschnittkomponente durchgeführt (Schmitt-Beck et al. 2010a).

Bei der Stichprobenziehung der Querschnitte findet zudem ein Oversampling von Befragten aus Ostdeutschland statt, sodass jeder dritte Befragte aus Ostdeutschland stammt. Damit zielt die GLES darauf ab, die in Ostdeutschland besonders zu erwartenden Veränderungen im Wahlverhalten auf der Grundlage ausreichender Fallzahl beobachten zu können (Schmitt-Beck et al. 2010a). Grundlegend geht die klassische Wahlforschung zwar davon aus, dass kurzfristige Faktoren an Einfluss gewinnen. Dennoch wirken auch heute noch langfristig erworbene Faktoren wie Parteiidentifikation oder das Vertrauen in politische Institutionen auf die Wahlentscheidung. Inwiefern diese Faktoren bei Bürger/innen aus dem östlichen Teil der Republik anderes wirken als im westlichen Teil, kann daher aufgrund des Oversamplings im östlichen Teil sowohl mittels Daten der Querschnitte als auch des Langfrist-Panels tiefgehend analysiert werden (vgl. hierzu bspw. Rohrschneider et al. 2012; Schoen 2009b).

Die Querschnitt- und Langfrist-Paneldaten werden mittels persönlich-mündlicher Befragungen mit ADM-Stichprobenziehung erhoben. Das bedeutet, dass die Interviewer/innen zu den Befragten nach Hause kommen und dort das Interview mit vorab definierten Haushaltsmitgliedern durchführen (Rattinger et al. 2012a). Erst wenn alle Zielpersonen der Stichprobe erreicht wurden, können die Daten bereinigt und zu einem analysierbaren Datensatz zusammengespielt werden. Dadurch ist es nicht machbar, anhand der Querschnitte die Effekte kurzfristiger Ereignisse zu erfassen oder kurzfristig mit der Integration neuer Fragen in das Frageprogramm zu reagieren.

Diesem Anliegen begegnet die Komponente 2 der GLES mittels einer inzwischen international etablierten Erhebungsmethode: der sogenannten RollingCross-Section (RCS) (ZA5302; Schmitt-Beck et al. 2010b). Dies ist eine telefonische Befragung, bei der eine Querschnitt-Zufallsstichprobe in kontrollierter Weise über einen zuvor definierten Zeitraum gespreizt wird, so dass die Befragten jedes Tages eine Zufallsstichprobe aus der Gesamtheit darstellen (Schmitt-Beck et al. 2010b, 1; Rattinger et al. 2011b). So können Trends der öffentlichen Meinung im Wahlkampf analysiert oder einzelne Erhebungstage in Verbindung mit bestimmten Ereignissen und/oder Personen betrachtet werden (vgl. hierzu bspw. Krewel et al. 2011; Schmitt-Beck 2011; Schmitt-Beck/Tenscher 2008; Schoen 2010). Eine Nachwahl-Panel-Komponente rundet die RCS ab. Dadurch können nicht nur Veränderungen auf Aggregatdatenebene, sondern auch auf Individualebene festgestellt werden (vgl. hierzu bspw. Partheymüller/Schmitt-Beck 2012; Schmitt-Beck/Partheymüller 2012; Schmitt-Beck et al. 2010b). Sollten unvorhergesehene Ereignisse geschehen, ist es aufgrund der tagesaktuellen Stichprobenziehung möglich, kurzfristig neue Fragen zu implementieren und dadurch die aktuellsten Trends und Stimmungen zu erfassen. Allerdings muss auch erwähnt werden, dass die tägliche Stichprobengroße mit 100 Befragten relativ klein und somit vornehmlich dazu geeignet ist, Veränderungen über die Zeit aufzuzeigen. Bei größeren Modellberechnungen ist es zu empfehlen, mit kumulierten tages- 
aktuellen Stichproben zu arbeiten, insbesondere in Anbetracht dessen, dass die Ausschöpfungsquoten bei Telefonstichproben hinsichtlich des Bildungsniveaus der Befragten teilweise stark verzerrt sind.

Zur Betrachtung der kurzfristigen Änderungen infolge von Wahlkampfdynamiken bietet sich zudem das Wahlkampf-Panel (Komponente 3, ZA5305) der GLES an. In diesem durch Onlinebefragungen realisierten Panel werden beginnend zwölf Wochen vor der Wahl zu sechs Zeitpunkten die gleichen Personen erneut befragt. Eine abschließende, siebte Befragung nach der Wahl rundet das Panel ab (Rattinger et al. 2012d; Schmitt-Beck et al. 2010a). Wie auch in der RCS-Befragung - allerdings durchgehend auf individueller Ebene - ist es dadurch möglich, Faktoren und Ereignisse zu identifizieren, die die Wahlentscheidung während des Wahlkampfs zur Bundestagswahl beeinflusst haben. Dadurch können nicht nur langfristige Änderungsprozesse, wie mittels des Langfrist-Panels operationalisierbar, sondern auch sehr kurzfristige Änderungen der Wähler/innenmeinung auf individueller Ebene identifiziert werden (vgl. hierzu bspw. Blumenstiel/Fürnberg 2012; Plischke/Bergmann 2012). Das Instrument der Online-Erhebung ermöglicht es ebenso kurzfristig auf neue Ereignisse durch das Implementieren neuer Fragen einzugehen. Allerdings ist anzumerken, dass Online-Stichproben große Probleme hinsichtlich ihrer Repräsentativität haben, was nachfolgend noch detaillierter ausgeführt wird.

\subsection{Herausforderung II: Personalisierung der Politik}

Wenn von der zunehmenden Orientierung an kurzfristigen Faktoren bei der Wahlentscheidung die Rede ist, so ist dies neben der Sachthemenorientierung insbesondere auf eine verstärkte Kandidatenorientierung bzw. Personalisierung der Wahlkämpfe seitens der Parteien bezogen (Schmitt-Beck 2011; Wagner/Weßels 2012b), die seit den 1990er Jahren auch verstärkt in Deutschland diagnostiziert wird (Brettschneider 2002). Daher ist es nicht überraschend, dass die GLES einen besonderen Schwerpunkt auf die intensive Erfassung der Kandidat/innen - seien es nun die Kanzlerkandidat/innen, die Spitzenkandidat/innen der Parteien oder die Wahlkreis- oder Landeslistenkandidat/innen - im Studiendesign legt. In allen Umfragekomponenten werden Fragen zu der Einschätzung der Spitzenkandidat/innen und der beiden Kanzlerkandidat/innen gestellt. Ebenso werden diese in den beiden Medieninhaltsanalysen entsprechend berücksichtigt. Der Vorwahl-Querschnitt geht noch einen Schritt weiter und erfasst, ob die Befragten ihre Wahlkreiskandidat/innen kennen und wie sie diese einschätzen (Rattinger et al. 2011c). Dadurch ist es möglich, nicht nur die führenden Politiker/innen und deren Wahlkämpfe zu betrachten, sondern auch einen Blick auf die Wahlkämpfe der Wahlkreiskandidat/innen und deren Effekte zu werfen (vgl. hierzu Gschwend/Zittel 2011; Schmitt-Beck 2011; Wagner/Weßels 2012a; 2012b).

Dieser Teil des Vorwahl-Querschnitts ist gekoppelt an Komponente 6, die Kandidatenstudie (ZA5318, ZA5319). Hier werden alle Wahlkreis- und Listenbewerber/innen zum Deutschen Bundestag nach der Bundestagswahl aufgefordert an einer schriftlichen Befragung bzw. Online-Befragung teilzunehmen und Auskunft über ihren politischen Hintergrund, ihre politischen Aktivitäten, 
ihre Wahlkampfführung und ihre Einstellung zu politischen Themen zu geben (Rattinger et al. 2012e). Dadurch ist es nicht nur möglich, die Sicht der Wähler/innen auf die Kandidat/innen zu erfassen, sondern auch Einblicke in die Welt der Wahlbewerber/innen zu erhalten. Dies ermöglicht tiefgehende Analysen ihrer Situation und ihrer Erfolgschancen (vgl. hierzu bspw. Bieber 2013; Giebler/Wüst 2011a; Gschwend/Zittel 2011; Metag/Marcinkowski 2012). Zudem beinhaltet diese Komponente einen Kernfragebogen des Comparative Candidates Surveys (CCS), der bisher in 19 weiteren Ländern in ähnlicher Weise abgefragt wird und damit die internationale Vernetzung der GLES stärkt (CCS 2013a; vgl. hierzu bspw. Giebler/Wüst 2011b; Schmitt-Beck et al. 2010a). Kritisch kann hierbei die zunächst durchgeführte Datenpolitik des CCS angemerkt werden: Zwar konnten die Daten der Deutschen Kandidatenstudie problemlos über die GESIS angefordert werden. Jedoch hatten die direkten Datenlieferanten einen privilegierten Zugriff auf die gesammelten Datensätze aller Länder. Dies hat sich seit kurzem geändert. Es wurde eine öffentlich zugängliche Datendistribution über FORS (Swiss Center of Expertise of the Social Science) eingerichtet, bei der aktuell Datensätze von 17 Wahlen in 15 Ländern angefordert werden können (CCS 2013b, FORS 2013).

Obwohl bei Bundestagswahlen nicht direkt der Kanzler bzw. die Kanzlerin gewählt werden kann, geraten zunehmend auch in Deutschland die beiden Kanzlerkandidat/innen in das Blickfeld der Aufmerksamkeit. Insbesondere gewinnen die sogenannten TV-Duelle seit Ende der 1990er Jahren an Beliebtheit und wurden zum ersten Mal auf Bundesebene 2002 mit Gerhard Schröder als Amtsinhaber und Edmund Stoiber als Herausforderer durchgeführt. Maier und Faas bezeichnen TV-Duelle sogar als „Wahlkampf in Miniaturformat“ (Maier/Faas 2005, 77). So sei es wahlentscheidend, wie die Spitzenkandidat/innen an diesem Abend argumentieren und welchen Eindruck sie bei den Wähler/innen hinterlassen. Den Einfluss eines Medienereignisses auf die Wahlentscheidung nachzuweisen ist nicht einfach, weshalb sich die Komponente 5 der GLES der TV-Duell-Analyse mittels der Durchführung eines vielfältigen Experiments widmet (Komponente 5; ZA5309, ZA5310, ZA5311, ZA5312, ZA5313, ZA5314, ZA5315, ZA5316): Diese Komponente baut auf Studien aus 2002 und 2005 auf und ermöglicht dadurch auch langfristig vergleichende Analysen (Maier/Faas 2011a). Das TV-Duell wird den Versuchspersonen in einem Hörsaal live übertragen. Dabei werden in einigen Versuchsgruppen sogenannte RTR-Messungen durchgeführt: Die Versuchspersonen haben dabei ein Messgerät, mittels dem sie während des gesamten TV-Duells angeben sollen, wie sie die beiden Kandidaten aktuell bewerten. Vor und nach dieser RTR-Messung werden die Teilnehmer/innen befragt. Ebenso findet einige Tage nach dem TV-Duell und kurz vor bzw. nach dem Wahltag eine weitere Befragung statt. Eine zusätzliche Inhaltsanalyse der TV-Debatte rundet Komponente 5 ab (Faas/Maier 2011; Rattinger et al. 2011d). Dadurch ist es möglich zu messen, ob und inwiefern die einzelnen Aussagen der Kanzlerkandidat/in auf die Wähler/innen kurz- und langfristig einen positiven oder negativen Effekt ausüben. Gekoppelt mit den Umfragedaten kann auch die Wirkung der Aussagen gruppenspezifisch betrachtet werden: Wird beispielsweise eine spezifische Aussage des SPD-Kandidaten von SPD-Anhänger/innen positiver bewertet als 
von Unions-Anhänger/innen (vgl. hierzu Maier/Faas 2011b)? Trotz dieser umfangreichen Erfassung der Wirkung des TV-Duells auf die Wähler/innenmeinung ist kritisch anzumerken, dass das TV-Duell nur ein Erklärungsfaktor von zahlreichen anderen Beeinflussungsmöglichkeiten im Wahlkampf ist und daher nicht final geklärt werden kann, welche Wirkungskraft die TV-Duelle haben. Ferner beschränken sich bisherige TV-Duell-Analysen zumeist darauf, die Entwicklungen der RTR-Messung in aggregierter Form zu betrachten und dabei die individuellen Unterschiede nur rudimentär zu berücksichtigen (vgl. hierzu Bachl/Vogelgesang 2013).

\subsection{Herausforderung III: Erfassung des elektoralen Kontextes}

Vor der GLES beschränkte sich die deutsche Wahlforschung darauf, direkt vor und/oder nach Bundestagswahlen große persönlich-mündliche oder telefonische Befragungen durchzuführen. Vereinzelt fanden zwischen den Wahlen sogenannte ,Zwischenerhebungen' vornehmlich zur Panelpflege statt. Um die Zeiten zwischen den Wahlen analysieren zu können, musste die wissenschaftliche Disziplin beispielsweise auf Daten der Forschungsgruppe Wahlen zurückgreifen, die für das Politbarometer des ZDF Umfragedaten in regelmäßigen Abständen produzieren und der Forschungsgemeinschaft zeitversetzt zur Verfügung stellen (GESIS 2013; Wüst 2003). Obwohl diese Daten, insbesondere durch die Kontinuität seit 1977, für die deutsche Wahlforschung bedeutend sind, ist die wissenschaftliche Disziplin hierbei vom Wohlwollen der Primärforscher abhängig und hat keinen Einfluss auf die Gestaltung der Erhebungen. Dies führt zu Einbußen in der Kontinuität einiger Erhebungsinstrumente. Zudem muss verhältnismäßig lange auf die Veröffentlichung der Daten gewartet werden. Diesen Defiziten begegnet die GLES durch das Langfrist-Online-Tracking (Komponente 8, ZA5334 bis ZA5351, ZA5719). Hier werden meist vierteljährlich Online-Befragungen durchgeführt, die sich aus einem kontinuierlichen Kernfrageteil, einem ausdifferenzierten Modulteil und tagesaktuellen Elementen zusammensetzen (vgl. bspw. Rattinger et al. 2013b; 2011e). In der Online-Tracking-Komponente wird der wissenschaftlichen Gemeinschaft ferner die Möglichkeit geboten, selbst Fragen in das Frageprogramm zu implementieren: Hierzu wurden bislang zwei Call for Questions mit der Bitte um Einsendung innovativer und vielversprechender Fragen bzw. Fragenblöcke durchgeführt (GLES 2013c). Dadurch soll die Offenheit und Rückkopplung an die wissenschaftliche Disziplin bestärkt werden. Neben der Analyse langfristiger Trends bietet sich das Online-Tracking auch dazu an, bisher aufgrund mangelnder Daten wenig erforschte Themengebiete zu untersuchen (vgl. hierzu Marschall/Schultze 2012; Römmele/Einwiller 2012; Scherer/Bieber 2013; Schultze 2012). Zu erwähnen ist hierbei außerdem, dass im Vorfeld der Bundestagswahl 2009, mit finanzieller Unterstützung von GESIS, sechs weitere Online-Trackings durchgeführt wurden, um einerseits Pretests im Rahmen der anderen Befragungskomponenten zu realisieren und andererseits verschiedene Themenschwerpunkte vor der Bundestagswahl zu erfassen und zudem einige experimentelle Designs zu realisieren (vgl. bspw. Rattinger et al. 2011f; 2011g). 
Neben den vierteljährlichen Tracking-Erhebungen beheimatet die GLES auch sogenannte Landtagswahlen-Boosts. Hier werden anlässlich von Landtagswahlen 500 Personen aus dem betreffenden Bundesland zu der Landtagswahl und bundespolitischen Themen befragt. Dies ermöglicht neben der intensiven Analyse des Wahlverhaltens anlässlich der Bundestagswahl auch einen Blick auf die Landesebene und den dort spezifisch wirkenden Prozessen (Rattinger et al. 2010, 2011h; 2013c; vgl. hierzu Blumberg/Kulick 2010; Onken/Schneider 2012). Da die Online-Tracking- und die LTW-Boost-Erhebungen zumeist parallel stattfinden, ist eine vergleichende Analyse der unterschiedlichen Wähler/innenmobilisierung und -meinung auf Bundes- und Landesebene möglich. Allerdings ist die Stichprobengröße von 500 Personen zu kritisieren, die zu Problemen bei der Modellierung einiger Fragestellungen führt. Ferner ist auch bei dieser Online-Komponente auf die Stichprobenproblematik hinzuweisen, die nachfolgend noch diskutiert wird.

\subsection{Herausforderung IV: Mediatisierung}

Die Beschreibung der Herausforderungen hat zudem gezeigt, dass die Präsenz der Medien größer wird und durch die Medienberichterstattung auch der Wahlentscheidungsprozess der Bürger/innen beeinflusst werden kann. Für Politiker/innen und Parteien wird es immer wichtiger, sich angemessen in den Medien zu präsentieren, um dadurch Einfluss auf die Wahlentscheidung der Bürger/innen und somit den Ausgang der Wahl zu nehmen (Brettschneider 2005). Die Rezeption von politikrelevanten Medieninhalten beeinflusst daher die Meinung, Einstellung und Verhaltensweise der Wähler/innen (Lau/Redawsk 2006). Damit derartige Analysen durchgeführt werden können beheimatet die GLES zwei Komponenten, die sich der Erhebung von Mediendaten widmen: Eine WahlkampfMedieninhaltsanalyse (Komponente 4, ZA5306, ZA5307), die die Kampagnen, Parteien und Kandidat/innen vor der jeweiligen Bundestagswahl im Fernsehen und Printmedien erfasst, bietet einerseits die Möglichkeit, die Entwicklung des Wahlkampfs in den Medien zu betrachten. Anderseits ist sie auch daraufhin angelegt, in Einklang mit der Rolling-Cross-Section die Wirkungen bestimmter Medienereignisse auf die Wähler/innenmeinung komponentenübergreifend zu erfassen (Schmitt-Beck et al. 2010a). Durch die tagesaktuelle Erhebung der öffentlichen Meinung durch die RCS und die Medieninhaltsanalyse ist es möglich zu prüfen, inwiefern verschiedene Themen und Ereignisse auf aggregierter Ebene tatsächlich zu Veränderungen auf der Wählerebene geführt haben (Schmitt-Beck 2012b; 2012c; vgl. hierzu bspw. Boomgaarden/Semetko 2012).

Neben dieser Wahlkampf-Medieninhaltsanalyse zeichnet sich die LangfristMedienagenda-Analyse (Komponente 9, ZA5323) durch eine quantitative computergestützte Inhaltsanalyse aus, die es ermöglicht, über einen langen Zeitraum die Medienagenda hinsichtlich politisch relevanter Ereignisse und der Bewertung von Parteien und Spitzenpolitiker/innen mittels der Häufigkeit der Nennung relevanter Begriffe in verschiedenen Medien zu erfassen (Schmitt-Beck et al. 2010a; Rattinger et al. 2013a). Die Basis hierzu bildet ein ausgefeiltes Diktionär, welches auf der Grundlage des Kategorienschemas der Wahlkampf-Medien- 
inhaltsanalyse auf- und ausgebaut wurde. ${ }^{2}$ Es werden kontinuierlich die politischen Berichte in den beiden Politikmagazinen Spiegel und Focus erhoben. Im Monat vor den Online-Trackings werden die politischen Berichte in den beiden überregionalen Tageszeitungen Frankfurter Allgemeinen Zeitung und Süddeutsche Zeitung und dem Boulevardblatt Bild erfasst, sodass die Interaktion zwischen Medienberichterstattung und Wähler/innenmeinung auch über die Zeit analysierbar ist (Komponente 8, ZA5534 bis ZA5719). Schließlich werden anlässlich der Landtagswahlen-Boosts politische Berichte in relevanten regionalen Zeitungen im Monat vor der Befragung erfasst. Dies ermöglicht die Analyse unterschiedlicher Medienberichterstattungen auf der Ebene der Nation und der Länder, um auch hier Mobilisierungseffekte nachweisen zu können. Dadurch ist es möglich, auch zwischen zwei Bundestagswahlen komponentenübergreifende Analysen hinsichtlich der Wirkung der Medienberichterstattung auf die Wähler/innenmeinung zu realisieren und einen Blick auf Nebenwahlen zu werfen.

\subsection{Herausforderung V: Stichprobenprobleme}

Das wohl größte Problem aller GLES-Befragungen mit Ausnahme der Kandidatenbefragung stellt sicherlich die Ziehung einer bevölkerungsrepräsentativen Stichprobe dar, auf deren Grundlagen valide Aussagen zu der Wähler/innenmeinung gemacht werden können. Grundlegend können hierbei zwei verschiedene Problemfelder identifiziert werden. Einerseits fällt die Ausschöpfungsquote bei den Stichproben der repräsentativen Querschnitts- und Telefonumfragen niedrig aus. Andererseits können mittels Online-Umfragen bevölkerungsrepräsentative Stichproben nur mit immensem Ressourcenaufwand realisiert werden.

Geringe Ausschöpfungsquoten haben einerseits zur Folge, dass mit einer Vielzahl an Personen, die mittels der Stichprobenziehung definiert wurden, kein Interview durchgeführt werden kann, da die Personen nicht zu erreichen sind oder sich nicht interviewen lassen wollen. Bei den Querschnitten belief sich 2009 die Ausschöpfungsquote auf 52,4 (Vorwahl) bzw. 51,4 Prozent (Nachwahl), bei der telefonischen Befragung auf 20 Prozent (Rattinger et al. 2011b; 2012a). Zahlreiche andere große Umfragen wie das SOEP oder die ALLBUS haben vergleichbare Schwierigkeiten bei der Realisierung hoher Ausschöpfungsquoten. Die GLES arbeitet derzeit an der Reduzierung dieses Problems, indem die Erfassung von Mobiltelefonanschlüssen in Telefonstichproben geprüft wird und im Querschnitt und dem Langfrist-Panel genaue Feldplanungen und -kontrollen die Ausschöpfungsquoten und die Wiederbefragungsbereitschaft möglichst hoch halten sollen. $\mathrm{Ob}$ und inwiefern diese Maßnahmen zu einer Verbesserung führen, ist derzeit noch unklar. Es ist allerdings zu erwarten, dass auch in der GLES - wie auch allen anderen Studien mit repräsentativen Bevölkerungsumfragen - diese Maßnahmen allein das Ausschöpfungsproblem nicht zufriedenstellend lösen können. Vielmehr ist davon auszugehen, dass diese Problematik weiterhin eine zentrale Herausforderung der GLES sein wird.

\footnotetext{
${ }^{2}$ Grundlage des Codierschemas für die Wahlkampf-Medieninhaltsanalyse und der LangfristMedienagenda-Analyse bilden Arbeiten von Roller 1991.
} 
Ein anderes Stichprobenproblem haben - wie bereits erwähnt - Online-Befragungen. Die Befragten wurden im Wahlkampf-Panel, in den Online-Trackings und den Landtagswahlen-Boosts der GLES bis 2012 aus einem sogenannten Online- bzw. Access-Panel von Respondi ausgewählt, bei dem sich jede Person selbst registrieren lassen kann, weshalb die Zusammensetzung des Panels nicht zufällig ist (ADM 2001). Dass hierbei keine repräsentative Stichprobe gezogen werden kann, liegt auf der Hand. Zudem ist davon auszugehen, dass sich die Qualität der Stichprobe insbesondere bei den Wahlkampf-Panelbefragungen mit zunehmender Zahl an Untersuchungswellen verschlechtern kann. Zwar konnte in Ansätzen nachgewiesen werden, dass Modelle der Wahlforschung gleichermaßen mit Online-Stichproben analysiert werden können, allerdings ist die Abbildung von deskriptiven Verteilungen - insbesondere in Bezug zur Wahlentscheidungsfrage - höchst problematisch und eine Überrepräsentation der Meinung von jüngeren und hochgebildeten Personen zu finden (vgl. hierzu Bieber/Bytzek 2012).

In Anbetracht dieser Stichprobenproblematik ist es nicht einfach, die gewünschte Trendentwicklung mittels der Online-Trackings darzustellen. Die Rekrutierung der Befragten mittels Online-Panels ist jedoch sehr kostengünstig und findet daher häufig Anwendung. Als die Methode der Wahl wird jedoch eine probability-based Befragtenrekrutierung gehandelt (Chang/Krosnick 2009; Couper/Miller 2008), bei der die Befragten auf der Grundlage einer tatsächlichen Zufallsstichprobe ausgewählt werden und ihnen ggf. noch Hard- und Software zur Verfügung gestellt werden, insofern die Personen bisher keinen Computer bzw. Internetanschluss besitzen. Diese Vorgehensweise ist jedoch sehr kostenintensiv. Um das Problem der Stichprobenziehung einerseits zu reduzieren, aber andererseits das Budget nicht zu sprengen, hat sich die GLES dazu entschieden, ab 2012 in den Online-Trackings und den Landtagswahlen-Boosts die Befragungsteilnehmer/innen aus einem zufällig telefonisch rekrutierten Offline-Panel von LINK auszuwählen (Rattinger et al. 2012f). Problematisch hierbei ist allerdings - wie bei telefonischen Befragungen häufig - die Überrepräsentativität von hochgebildeten Personen, die sich in entsprechender Weise ebenso im offlinerekrutierten LINK-Panel wiederfinden, weshalb auch hier mit quotierten Stichproben der Versuch unternommen wird, die Verzerrungen zu reduzieren (Rattinger et al. 2012f.). Ob dadurch tatsächlich eine verbesserte Abbildung der wahlberechtigten Bürger/innen realisiert werden kann und zukünftig ähnliche Trendanalysen wie etwa mittels der Politbarometer-Daten möglich sind, ist noch zu prüfen.

Grundsätzlich ist in Frage zu stellen, inwiefern die verschiedenen Komponenten der GLES (persönlich-mündliches Interview, Telefonbefragung, OnlineBefragung) - insbesondere aufgrund der Stichprobenproblematik und der daraus resultierenden sozialstrukturell unterschiedlichen Zusammensetzung der Stichprobe - bei der Auswertung tatsächlich miteinander verbunden werden können. Es ist davon auszugehen, dass Modeeffekte die Validität der Aussagen beeinflussen. Erste Analysen, die die verschiedenen Befragungsmodi der persönlichen, der Telefon- und der Online-Umfrage der Wahlforschung vergleichend analysiert haben, konnten jedoch bei klassischen Modellberechnungen der Wahlforschung mittels verschiedener Stichproben nachweisen, dass die Ergebnisse vergleichbar waren und somit auch mittels den als besonders problematisch geltenden Online- 
Stichproben multivariate Berechnungen zu vergleichbaren Ergebnissen kommen wie Berechnungen mittels Telefon- und persönlichen Befragungen.

Möglicherweise bietet sich gerade infolge der verschiedenen Befragungsmodi der GLES eine Möglichkeit an, der Stichprobenproblematik und den damit in Verbindung stehenden problematischen Ausschöpfungsquoten zu begegnen: In der Logik von Mixed-Mode-Umfragen (vgl. hierzu Dillman et al. 2008; Nigg et al. 2009; Revilla 2010), also der Zusammenstellung eines gemeinsamen Datensatzes aus verschiedenen Datenquellen mit unterschiedlichen Befragungsmodi, könnten die Probleme der einzelnen Erhebungsmethoden eventuell in der geeigneten Kombination kompensiert werden. Sicherlich kann dieser Datensatz nur gewisse Kernbereiche der empirischen Wahlforschung umfassen und es ist zu empfehlen derartige Verfahrensweise zuvor intensiv hinsichtlich Validität und Angemessenheit zu testen.

Bezüglich der Stichprobenproblematik ist folglich festzuhalten, dass dies auch zukünftig ein zentraler Bereich sein wird, in dem die GLES vor großen Herausforderungen steht, die es zu berücksichtigen gilt.

\subsection{Herausforderung VI: Herausforderung VI: Theoretische Defizite}

Ein inhärentes Problem der empirischen Wahlforschung stellt ein gewisser „Theorieeklektizismus" (Schoen 2009a) dar, der aus einem bislang fehlenden gemeinsamen Theoriengebäude besteht, aus dem heraus neue theoretische Ansätze problemlos entwickelt und rückgekoppelt werden können. Zudem sind in der empirischen Wahlforschung immer häufiger mikropsychologische Theorien der Entscheidungsfindung zu berücksichtigen. Aufgrund der Veränderungen bei den Wähler/innen und im elektoralen Kontext und der zunehmenden Dichte an politischen Informationen infolge der Mediatisierung und Professionalisierung politischer Kommunikation ist ein theoretischer Rahmen notwendig, der einerseits auf die Dynamiken und die Unterschiede im Wahlentscheidungsprozess eingeht und andererseits die strukturellen Änderungen infolge der Mediatisierung berücksichtigt. Das bedeutet also, dass sich die Wähler/innen hinsichtlich Einstellungen, Meinungen, politischem Interesse und kognitivem Leistungsvermögen unterscheiden und grundsätzlich nicht in der Lage sind, alle Informationen, die ihnen von den Kandidat/innen, Parteien und Medien vermittelt werden, aufzunehmen. Die internationale Forschung spricht in diesen Kontext von einer ,informationprocessing" Perspektive (Lau/Redlawsk 2006). Um trotz der Vielzahl an Informationen, partiellem Desinteresse und unterschiedlichem kognitivem Vermögen ihre Wahlentscheidung treffen können, benutzen die Wähler/innen Heuristiken bzw. sogenannte Short-Cuts.

Die GLES hat es sich grundsätzlich zur Aufgabe gemacht, eine solche information processing-Perspektive einzunehmen, bei der Informationsverarbeitungsprozesse der Wähler/innen genau betrachtet werden. In erster Linie ist zu erkennen, dass die GLES dieser Herausforderung bisher insbesondere im Rahmen der Ausgestaltung der verschiedenen Komponenten begegnet, welche in ihrer Vielfalt grundsätzlich zahlreiche Möglichkeiten bieten, diese Heuristiken und ShortCuts zu erfassen. Ferner ist die GLES bestrebt, gemeinsame Publikationen zu 
konzipieren und zu realisieren, die diesem Anspruch des gemeinsamen Theoriegebäudes unter Rückkopplung bisheriger theoretischer Ansätze genügen sollen. Allerdings ist hier zu wünschen, dass die GLES - und auch alle anderen Wahlforscher/innen - zukünftig diese Herausforderung in stärkerem Maße annehmen und entsprechende Abhandlungen hierzu verfassen, die dem Theorieneklektizismus stärker entgegentreten als dies bislang der Fall ist.

Ferner werden zu selten mikropsychologische Theorien der Entscheidungsfindung und Einstellungsbildung herangezogen, die zudem gesellschaftliche und politische Kontextfaktoren berücksichtigen. Auch dieses Problem bearbeitet die GLES derzeit insbesondere durch die Bereitstellung von Daten, die derartige Analysen ermöglichen: Es werden zunehmend die verschiedenen Ebenen des politischen Systems Deutschlands berücksichtigt und erfasst. Wie erwähnt, werden anlässlich von Landtagswahlen Online-Befragungen mit Personen aus dem jeweiligen Bundesland durchgeführt. Dies ermöglicht neben der intensiven Analyse des Wahlverhaltens anlässlich der Bundestagswahl auch einen Blick auf die Landesebene und den dort spezifisch wirkenden Prozessen (Rattinger et al. 2010; 2011h; 2013c; vgl. hierzu Blumberg/Kulick 2010; Onken/Schneider 2012). Ferner wurde 2009 - einem Jahr, indem sowohl die Bundestagswahl, Europawahl als auch in einigen Ländern Landtagswahlen stattfanden - ein sogenanntes MultiLevel-Panel durchgeführt. Hier wurden in den Ländern mit Landtagswahl (Brandenburg, Saarland, Sachsen, Schleswig-Holstein, Thüringen) und einer Kontrollgruppe jeweils 500 Personen zu drei Zeitpunkten (Mai/Juni, August, September) befragt, um individuelle Änderungsprozesse in Bezug zu den Wahlentscheidungen auf Bundes-, Landes- und Europaebene zu erfassen (vgl. hierzu Rattinger et al. 2011i). Eine Datenerhebungsmethode, die die Analyse des Einflusses der verschiedenen Ebenen auf die Wahlentscheidung ermöglicht. Allerdings ist auch hier die Gruppengröße von 500 Personen je Bundesland bzw. Kontrollgruppe und die Panelmortalität zu kritisieren, die bei der empirischen Analyse Schwierigkeiten bereitet.

Neben der Bereitstellung von Daten auf mehreren Ebenen reagiert die GLES auf die Berücksichtigung mikropsychologischer Theorien mit der Durchführung von Experimenten. Diese sind im Online-Modus technisch problemlos und kostengünstig zu realisieren. Es wurden verschiedene Experimente inhaltlicher und technischer Natur durchgeführt: Anlässlich der Bundestagswahl wurde beispielsweise ein Kandidatenexperiment realisiert (Bieber 2013), in Tracking 14 ein Experiment über die Wirkung von Framing-Effekten (Mader/Schoen 2013) oder über die Antwortreaktionszeiten der Befragten (Plischke 2012). Doch dies kann erst der Anfang experimenteller Studien in der Wahlforschung sein. Denn insbesondere die Online-Methode der Datensammlung bietet zahlreiche weitere Möglichkeiten experimentell das Wahlverhalten der Bürger/innen zu erfassen.

\section{Zusammenfassung und Fazit}

Wie aufgezeigt werden konnte, steht die deutsche Wahlforschung bedingt durch interne und externe Faktoren vor großen Herausforderungen: Gesellschaftliche 
Individualisierungsprozesse zwingen die Wahlforscher/innen zunehmend dazu, einen stärkeren Fokus auf kurzfristige Faktoren zu richten, was mittels der bisherigen Standarderhebungsmethode der Wahlforschung - einer Vor- und Nachwahlbefragung anlässlich der Bundestagswahl - nur noch in eingeschränktem Maße möglich war. Ebenso tragen Entwicklungen des Mediensystems und dadurch bedingt der Wahlkampfkommunikation dazu bei, neue Erhebungsinstrumente zu etablieren, um diese Prozesse erfassen und analysieren zu können. Ferner gilt es allgemeinen Problemen der Umfrageforschung, wie z.B. Stichproben- und Ausschöpfungsproblemen, zu begegnen und an theoretischen Defiziten zu arbeiten.

Diesen Herausforderungen begegnet die empirische Wahlforschung insbesondere in der GLES, der sogenannten Deutschen Wahlstudie. Insgesamt neun Komponenten mit unterschiedlichen Erhebungsmethoden und Schwerpunktsetzungen erheben Daten, die sowohl die Analyse kurzfristiger als auch langfristiger Einflussfaktoren, den Einfluss von Medien bzw. Medienereignissen, der Parteien, der Kandidat/innen oder TV-Duellen auf individueller und aggregierter Ebene ermöglichen. Die GLES berücksichtigt in der Instrumentenentwicklung und Fragebogenformulierung stets die vorherigen Erhebungen der deutschen Wahlforschung und dockt sich gleichfalls an internationale Studien an, sodass eine kontinuierliche und international vergleichende Forschung möglich ist. Bedeutend ist ebenso, dass die Datenerhebung nicht nur die Zeit vor und nach einer Bundestagswahl erfasst, sondern durch das Online-Tracking und die LangfristMedienagenda-Analyse eine kontinuierliche Datenerhebung sichergestellt ist, die ebenso die Analyse langfristiger Entwicklungen und Medienereignisse ermöglicht. Die interne Abstimmung der Studienkomponenten hinsichtlich gemeinsamen und individuellem komponentenspezifischem Fragenprogramm trägt einerseits zu intern vergleichenden bzw. kompatiblen Analysen und andererseits zur Bereitstellung von Daten für spezifische Fragestellungen bei. Dies wird in besonderem Maße durch das Online-Tracking verwirklicht, da hier häufig Erhebungen stattfinden, in denen auch Daten zur Analyse weniger etablierter Theorien erhoben werden können.

An dieser Stelle ist zu betonen, dass es der GLES gelungen ist, in den vergangenen Jahren die verschiedenen Komponenten zu konzipieren, zu realisieren und eine Vielzahl an Daten zu erheben. Ebenso ist darauf hinzuweisen, dass alle Daten bereits kurz nach der Erhebung der wissenschaftlichen Gemeinschaft (distribuiert durch GESIS) zur Verfügung gestellt werden, um zeitnah Forschungsarbeiten aller Wissenschaftler/innen zu ermöglichen und dadurch ein immenses Kollektivgut zu produzieren. Der 10.407fache Download von GLES-Datensätzen zeigt auch den Erfolg der Studie (Stand: 31. August 2013).

Doch nicht allen Herausforderungen der empirischen Wahlforschung konnte die GLES bislang zur Genüge begegnen. Auch sie hat - wie zahlreiche andere Umfragen - Probleme bei der Ziehung bevölkerungsrepräsentativer Stichproben aufgrund niedriger Ausschöpfungsquoten. Ebenso ist die Stichprobenproblematik bei Online-Umfragen anzumerken, die ohne Quotierung junge und hochgebildete Personen überrepräsentiert. Auf diese Herausforderung wird die GLES zukünftig Antworten finden müssen, um rechtzeitig Gegenmaßnahmen ergreifen zu können. 
Bezüglich der Herausforderungen der Wahlforschung im Bereich des ,theoretischen Defizits' muss an dieser Stelle ferner festgehalten werden, dass die GLES hierzu bislang nicht zufriedenstellende Antworten angeboten hat. Zwar stellt die GLES massenhaft Daten zur Analyse dieser Herausforderungen bereit, jedoch fehlt es bislang an theoretischem Fundament. Auf der anderen Seite ist jedoch auch festzuhalten, dass es nicht alleinige Aufgabe der GLES ist, alle Herausforderungen der empirischen Wahlforschung zu lösen. So ist auch die Gemeinschaft aller Wahlforscher/innen gefragt, theoretische Antworten auf die Modernisierungsprozesse im Wahlverhalten und der politischen Kommunikation zu finden, an der theoretischen Weiterentwicklung zu arbeiten und adäquate empirische Lösungsstrategien anzubieten.

So ist zu erwarten, dass die empirische Wahlforschung, nachdem nun mit der GLES vielfältige Erhebungsinstrumente in der deutschen Forschung etabliert und zunehmend standardisiert sind, sich zukünftig in noch verstärktem Maße den verschiedenen theoretischen Defiziten widmen kann und an der theoretischen Rückkopplung empirischer Analysen - insofern dies noch nicht geschehen ist - arbeiten kann. Die GLES bietet somit eine gute Ausgangsbasis für eine positive Entwicklung der empirischen Wahlforschung in Deutschland und im internationalen Vergleich.

\section{Bibliographie}

Arbeitskreis Deutscher Markt- und Sozialforschungsinstitute (ADM) (2001), Standards zur Qualitätssicherung für Online-Befragungen, URL: http://www.adm-ev.de /fileadmin/user_upload/PDFS/Onlinestandards_D.PDF [30.5.2011]

Arzheimer, K./A. Schmitt (2005), Der ökonomische Ansatz, in: Falter, J. W./H. Schoen (Hg.), Handbuch Wahlforschung, Wiesbaden, 243-303

Bachl, M./ J. Vogelgesang (2013), Analyzing Continuous Response Measurement Data Using Cross-classified Growth Curve Models, Vortrag auf der 63. Jahrestagung der International Communication Association, 17.-21.6.2013, London

Beck, U. (1983), Jenseits von Stand und Klasse? Soziale Ungleichheit, gesellschaftliche Individualisierungstendenzen und die Entstehung neuer sozialer Formationen und Identitäten, in: Kreckel, R. (Hg.), Soziale Ungleichheiten, Göttingen, 35-74

Behnke, J. (2008), Strategisches Wählen bei der Nachwahl in Dresden zur Bundestagswahl 2005, in: Politische Vierteljahresschrift 49, 695-720

Bieber, I. E. (2013), Frauen in der Politik. Einflussfaktoren auf weibliche Kandidaturen zum Deutschen Bundestag, Wiesbaden

-/E. Bytzek (2012), Online-Umfragen: Eine geeignete Erhebungsmethode für die Wahlforschung? Ein Vergleich unterschiedlicher Befragungsmodi am Beispiel der Bundestagswahl 2009, in: Methoden - Daten - Analysen 6, 185-211

Blumenberg, J./M. Kulick (2010), Der geliebte Verräter. Zum Einfluss von Spitzenkandidaten auf das Wahlverhalten am Beispiel der saarländischen Landtagswahl 2009, in: Zeitschrift für Parlamentsfragen 41, 803-817

Blumenstiel, J. E./O. Fürnberg (2012), Wissen und Einstellungen zu Überhangsmandaten, in: Zeitschrift für Parlamentsfragen 1, 132-141 
Boomgaarden, H. G./H. A. Semetko (2007), Duell Mann gegen Frau?! Geschlechterrollen und Kanzlerkandidaten in der Wahlkampfberichterstattung, in: Brettschneider, F./O. Niedermayer/B. Weßels (Hg.), Die Bundestagswahl 2005. Analysen des Wahlkampfes und der Wahlergebnisse, Wiesbaden, 171-196

-/_ (2012), Nachrichten-Bias: Medieninhalte, Bevölkerungswahrnehmungen und Wahlentscheidungen bei der Bundestagswahl 2009, in: PVS-Sonderheft 45 (Wählen in Deutschland), 442-464

Brettschneider, F. (2002), Spitzenkandidaten und Wahlerfolg. Personalisierung, Kompetenz, Parteien. Ein internationaler Vergleich, Wiesbaden

- (2005), Massenmedien und Wählerverhalten, in: Falter, J. W./H. Schoen (Hg.), Handbuch Wahlforschung, Wiesbaden, 473-500

-/M. Rettich (2005), Medieneinflüsse auf das Wahlverhalten, in: Falter, J. W./O. W. Gabriel/B. Weßels (Hg.), Wahlen und Wähler: Analysen aus Anlass der Bundestagswahl 2002, Wiesbaden, 157-185

Campbell, A./P. E. Converse/W. E. Miller/D. E. Stokes (1960), The American Voter, New York

CCS (Comparative Candidates Survey) (2013a), The Comparative Candidates Survey -Participating Countries, URL: http://www.comparativecandidates.org/node/3 [1.8.2013]

- (2013b), The Comparative Candidates Survey-Participating Countries, URL: http://www.comparativecandidates.org/node/1 [1.8.2013]

Chang, L./J. A. Krosnick (2009), National Surveys via RDD Telephone Interviewing versus the Internet. Comparing Sample Representativeness and Response Quality, in: Public Opinion Quarterly 73, 641-678

Clark, T. N./S. Lipset (1991), Are Social Classes Dying?, in: International Sociology 6, 397-410

Couper, M. P./P. V. Miller (2008), Web Survey Methods, in: Public Opinion Quarterly $72,831-835$

Dalton, R. J. (2012), Apartisans and the Changing German Electorate, in: Electoral Studies 31, 35-45

Debus, M. (2012), Sozialstrukturelle und einstellungsbasierte Determinanten des Wahlverhaltens und ihr Einfluss bei Bundestagswahlen im Zeitverlauf: Westdeutschland 1976 bis 2009, in: PVS-Sonderheft 45 (Wählen in Deutschland), 40-62

Dillman, D. A./J. D. Smyth/L. M. Christian (2008), Internet, Mail, and Mixed-Mode Surveys: The Tailored Design Method, New Jersey

Downs, A. (1957), An Economic Theory of Democracy, New York

Eder, C./M. Kulick/C. Wolf (2010), Daten, Service und Analysen für die Wahlforschung, in: Zeitschrift für Politikberatung 3, 217-222

Elff, M. (2007), Social Structure and Electoral Behavior in Comparative Perspective: The Decline of Social Cleavages in Western Europe Revisited, in: Perspectives on Politics 5, 277-294

- S. Roßsteutscher (2011), Stability or Decline? Class, Religion and the Vote in Germany, in: German Politics 20, 107-127

Faas, T./S. Huber (2010), Experimente in der Politikwissenschaft: Vom Mauerblümchen zum Mainstream, in: Politische Vierteljahresschrift 51, 721-749

-/K. Arzheimer/S. Roßteutscher (2010), Information - Wahrnehmung - Emotion, in: Faas, T./K. Arzheimer/S. Roßteutscher (Hg.), Information - Wahrnehmung - Emotion. Politische Psychologie in der Wahl- und Einstellungsforschung, Wiesbaden, $7-12$ 
-/J. Maier (2011), Medienwahlkampf: Sind TV-Duelle nur Show und damit nutzlos?, in: Bytzek, E./S. Roßteutscher (Hg.), Der unbekannte Wähler? Mythen und Fakten über das Wahlverhalten der Deutschen, Frankfurt a.M.-Wiesbaden, 99-114

Falter, J. W./H. Schoen/C. Caballero (2000), Dreißig Jahre danach. Zur Validierung des Konzepts ,Parteiidentifikation‘ in der Bundesrepublik, in: Klein, M./W. Jagodzinski/E. Mochmann/D. Ohr (Hg.), 50 Jahre empirische Wahlforschung in Deutschland. Entwicklung, Befunde, Perspektiven, Daten, Wiesbaden, 235-271

-/_ (2005) (Hg.), Handbuch Wahlforschung, Wiesbaden

Fiorina, M. P. (1981), Retrospective Voting in American National Elections, New Haven

FORS (2013): DARIS - Daten und Forschungsinformationsservice, Comparative Candidate Survey (CCS), URL: http://www2.unil.ch/daris/spip.php?rubrique157 \&lang $=$ de [23.9.2013]

Gabriel, O. W./S. I. Keil (2005), Empirische Wahlforschung in Deutschland: Kritik und Entwicklungsperspektiven, in: Falter, J. W./H. Schoen (Hg.), Handbuch Wahlforschung, Wiesbaden, 611-641

GESIS (2013), Politbarometer (1977-2011), URL: http://www.gesis.org/wahlen/ politbarometer/ [28.5.13]

GLES (2013a), Website der German Longitudinal Election Study, URL: http://www. gles.eu/index.htm [28.5.2013]

- (2013b), Website der German Longitudinal Election Study, URL: http://www.gles. eu/design.htm [26.09.2013]

- (2013c), Call for Questions zum GLES-Online-Tracking, URL: http://www.gles.eu /Cfq_dt.pdf [28.5.13]

Giebler, H./A. Wüst (2011a), Individuelle Wahlkämpfe bei der Europawahl 2009: Länderübergreifende und ebenenspezifische Befunde, in: Tenscher, J. (Hg.), Superwahljahr 2009. Vergleichende Analysen aus Anlass der Wahlen zum Deutschen Bundestag und zum Europäischen Parlament, Wiesbaden, 121-152

- / - (2011b), Campaigning on an Upper Level? Individual Campaigning in the 2009 European Parliament Elections in Its Determinants, in: Electoral Studies 30, 53-66

Gschwend, T. (2007), Ticket-splitting and Strategic Voting under Mixed Electoral Rules. Evidence from Germany, in: European Journal of Political Studies 46, 1-23

-/T. Zittel (2011), Machen Wahlkreiskandidaten einen Unterschied? Die Persönlichkeitswahl als interaktiver Prozess, in: PVS-Sonderheft 45 (Wählen in Deutschland), 371-392

Häder, M. (2011), Empirische Sozialforschung, Wiesbaden

Herrmann, M./F. U. Pappi (2008), Strategic Voting in German Constituencies, in: Electoral Studies 27, 228-244

Hunsicker, S./Y. Schroth (2007), Die Kombination von Mobilfunk- und Festnetzstichproben, in: Methoden - Daten - Analysen 1, 161-182

Kaase, M. (2000), Entwicklung und Stand der Empirischen Wahlforschung in Deutschland, in: Klein, M./W. Jagodzinski/E. Mochmann/D. Ohr (Hg.), 50 Jahre empirische Wahlforschung in Deutschland. Entwicklung, Befunde, Perspektiven, Daten, Wiesbaden, $17-40$

Kohler, U. (2005), Statusinkonsistenz und Entstrukturierung von Lebenslagen. Empirische Untersuchung zweier Individualisierungshypothesen mit Querschnittsdaten aus 28 Ländern, in: Kölner Zeitschrift für Soziologie und Sozialpsychologie 57, 230253 
Krewel, M./R. Schmitt-Beck/A. Wolsing (2011), The Campaign and Its Dynamics at the 2009 German General Election, in: German Politics 20, 28-50

Lachat, R. (2007), A Heterogeneous Electorate. Political Sophistication, Predisposition Strength, and the Voting Decision Process, Baden-Baden

Lau, R. R./D. P. Redlawsk (2006), How Voters Decide. Information Processing during Election Campaigns, Cambridge

Lazarsfeld, P./B. Berelson/H. Gaudet (1968), The People's Choice. How the Voter Makes Up His Mind in a Presidential Campaign, New York-London

Lipset, S. M./S. Rokkan (1967), Cleavage Structures, Party Systems, and Voter Alignments. An Introduction, in: Lipset, S. M./S. Rokkan (eds.), Party Systems and Voter Alignments, New York, 1-64

Mader, M./H. Schoen (2013), Alles eine Frage des Blickwinkels? Framing-Effekte und Bevölkerungsurteile über einen möglichen Bundeswehreinsatz in Libyen, in: Zeitschrift für Internationale Beziehungen 20, 5-34

Maier, J./M. Maier (2007), Das TV-Duell 2005: Katalysator für die Personalisierung des Wahlverhaltens?, in: Brettschneider, F./O. Niedermayer/B. Weßels (Hg.), Die Bundestagswahl 2005. Analysen des Wahlkampfes und der Wahlergebnisse, Wiesbaden, 219-232

—/T. Faas (2005), Schröder gegen Stoiber: Wahrnehmung, Verarbeitung und Wirkung der Fernsehdebatten im Bundestagswahlkampf 2002, in: Falter, J. W./O. W. Gabriel/B. Weßels (Hg.), Wahlen und Wähler: Analysen aus Anlass der Bundestagswahl 2002, Wiesbaden, 77-101

-/_ (2011a), 'Miniature Campaigns' in Comparison: The German Televised Debates, 2002-09, in: German Politics 20, 75-91

—/_ (2011b), Das TV-Duell 2009 - langweilig, wirkungslos, nutzlos? Ergebnisse eines Experiments zur Wirkung der Fernsehdebatte zwischen Angela Merkel und FrankWalter Steinmeier, in: Oberreuter, H. (Hg.), Am Ende der Gewissheiten: Wähler, Parteien und Koalitionen in Bewegung, München, 147-166

—/_/M. Maier (2013), Mobilisierung durch Fernsehdebatten: Zum Einfluss des TVDuells 2009 auf die politische Involvierung und die Partizipationsbereitschaft, in: Weßels, B./H. Schoen/O. W. Gabriel (Hg.), Wahlen und Wähler. Analysen aus Anlass der Bundestagswahl 2009, Wiesbaden, 79-96

Marschall, S./M. Schultze (2012), Normalisierung oder Mobilisierung? Die Auswirkungen politischer Online-Kommunikation auf die Wahlbeteiligung am Beispiel einer Internet-Applikation zur Bundestagswahl 2009, in: PVS 53, 444-466

Meffert, M. F./T. Gschwend (2010), Strategic Coalition Voting: Evidence from Austria, in: Electoral Studies 29, 339-349

Metag, J./F. Marcinkowski (2012), Strategic, Structural, and Individual Determinants of Online Campaigning in German Elections, in: Policy and Internet 4, 139-158

Müller, W. (1997), Sozialstruktur und Wahlverhalten. Eine Widerrede gegen die Individualisierungshypothese, in: Kölner Zeitschrift für Soziologie und Sozialpsychologie 49, $747-760$

- (1999), Class Cleavages and Party Preferences in Germany-Old and New, in: Evans, G. (ed.), The End of Class Politics? Class Voting in Comparative Context, Oxford, 137-180

-/M. Klein (2012), Die Klassenbasis in der Parteipräferenz des deutschen Wählers. Erosion oder Wandel?, in: PVS-Sonderheft 45 (Wählen in Deutschland), 85-110 
Niedermayer, O. (2007), Der Wahlkampf zur Bundestagswahl 2005: Parteistrategien und Kampagnenverlauf, in: Brettschneider, F./O. Niedermayer/B. Weßels (Hg.), Die Bundestagswahl 2005. Analysen des Wahlkampfes und der Wahlergebnisse, Wiesbaden, 21-42

Nigg, C. R./R. W. Motl/K. T. Wong/L. U. Yoda/D. K. McCurdy/R. Paxton/C. C. Horwath/R. K. Dishman (2009), Impact of Mixed Survey Modes on Physical Activity and Fruit/Vegetable Consumption: A Longitudinal Study, in: Survey Research Methods 3, 81-90

Noelle-Neumann, E. (2000), Der Beitrag der Wahlforschung zur Methodenentwicklung in der empirischen Sozialforschung, in: Klein, M./W. Jagodzinski/E. Mochmann/D. Ohr (Hg.), 50 Jahre empirische Wahlforschung in Deutschland. Entwicklung, Befunde, Perspektiven, Daten, Wiesbaden, 59-81

Ohr, D./M. Quandt (2012), Parteiidentifikation in Deutschland: Eine empirische Fundierung des Konzepts auf Basis der Theorie Sozialer Identität, in: PVS-Sonderheft 45 (Wählen in Deutschland), 179-202

Onken, H./S. H. Schneider (2012), Entern, kentern oder auflaufen? Zu den Aussichten der Piratenpartei im deutschen Parteiensystem, in: Zeitschrift für Parlamentsfragen 43, 609-625

Pappi, F. U. (1973), Parteiensystem und Sozialstruktur in der Bundesrepublik, in: Politische Vierteljahresschrift 14, 191-213

- (1977), Sozialstruktur, gesellschaftliche Wertorientierungen und Wahlabsicht. Ergebnisse eines Zeitvergleichs des deutschen Elektorats 1953 und 1976, in: Kaase, M. (Hg.), Wahlsoziologie heute. Analysen aus Anlass der Bundestagswahl 1976, Opladen, 195-229

- (1989), Räumliche Modelle in der Parteienkonkurrenz. Die Bedeutung ideologischer Dimensionen, in: Falter, J. W./H. Rattinger/K. G. Troitsch (Hg.), Wahlen und politische Einstellungen in der Bundesrepublik Deutschland, Frankfurt a.M., 5-28

-/S. Shikano (2001), Personalisierung der Politik in Mehrparteiensystemen am Beispiel deutscher Bundestagswahlen seit 1980, in: Politische Vierteljahresschrift 42, $355-387$

-/P. W. Thurner (2002), Electoral Behaviour in a Two-Vote-System: Incentives for Ticket Splitting in German Bundestag Elections, in: European Journal of Political Research 41, 207-232

-/S. Shikano (2007), Wahl- und Wählerforschung, Baden-Baden

Partheymüller, J./R. Schmitt-Beck (2012), A ,Social Logic' of Demobilization: The Influence of Political Discussants on Electoral Participation at the 2009 German Federal Election, in: Journal of Elections, Public Opinion and Parties 22, 457-478

Plischke, T. (2012), Reaktionszeiten als Indikatoren für politische Einstellungen- Der Implizite Assoziationstest (IAT), in: Methoden - Daten - Analysen 6, 73-98

-/M. Bergmann (2012), Entscheidungsprozesse von Spätentscheidern bei der Bundestagswahl 2009, in: PVS-Sonderheft 45 (Wählen in Deutschland), 489-513

Rattinger, H. (1998), Normalwahlanalyse monatlicher Parteipräferenzen in Westdeutschland von 1978 bis 1994, in: Kaase, M./H.-D. Klingemann (Hg.), Wahlen und Wähler. Analysen aus Anlass der Bundestagswahl 1994, Opladen, 357-390

-/S. Roßsteutscher/R. Schmitt-Beck/B. Weßels (2010), Landtagswahl NordrheinWestfalen 2010, ZA5324, Studienbeschreibung, GESIS Datenarchiv, Köln

$-/-/-/-/$ I. Bieber/J. E. Blumenstiel/E. Bytzek/T. Faas/S. Huber/M. Krewel/J. Maier/T. Rudi/P. Scherer/M. Steinbrecher/A. Wagner/A. Wolsing (2011a), Zwischen Langeweile und Extremen: Die Bundestagswahl 2009, Baden-Baden 
-/—/_/ (2011b), Rolling Cross-Section-Wahlkampfstudie mit Nachwahl-Panelwelle, ZA5303, Studienbeschreibung, GESIS Datenarchiv, Köln

-/-/-/— (2011c), Vorwahl-Querschnitt, ZA5300, Fragebogendokumentation, GESIS Datenarchiv, Köln

-/-/-/-/F. Brettschneider/T. Faas/J. Maier/M. Maier (2011d), TV-Duell-Analyse ZA5309, ZA5310, ZA5311, ZA5312, ZA5313, ZA5314, ZA5315, ZA5316, Studienbeschreibung, GESIS Datenarchiv, Köln

-/-/-/- (2011e), Langfrist-Online-Tracking, T6, ZA5339, Studienbeschreibung, GESIS Datenarchiv, Köln

-/-/-/Bernhard (2011f), Langfrist-Online-Tracking, T1, ZA5334, Studienbeschreibung, GESIS Datenarchiv, Köln

-/-/-/- (2011g), Langfrist-Online-Tracking, T2, ZA5335, GESIS Datenarchiv, Studienbeschreibung, GESIS Datenarchiv, Köln

-/-/-/— (2011h), Landtagswahl Mecklenburg-Vorpommern 2011, ZA5330, Studienbeschreibung, GESIS Datenarchiv, Köln

-/-/—/_ (2011i), Mulit-Level-Panel, ZA5304, Studienbeschreibung, GESIS Datenarchiv, Köln

-/-/-/- (2012a), Vor- und Nachwahl-Querschnitt, ZA5302, Studienbeschreibung, GESIS Datenarchiv, Köln

-/-/-/- (2012b), Langfrist-Panel 2002-2005-2009 ZA5320, Studienbeschreibung, GESIS Datenarchiv, Köln

-/-/-/- (2012c), Langfrist-Panel 2005-2009-2013, ZA5321, Studienbeschreibung, GESIS Datenarchiv, Köln

-/-/-/— (2012d), Wahlkampf-Panel, ZA5305, Studienbeschreibung, GESIS Datenarchiv, Köln

-/-/-/-/T. Gschwend/H. Schmitt/A. Wüst/T. Zittel (2012e), Kandidatenstudie, ZA5319, Studienbeschreibung, GESIS Datenarchiv, Köln

-/-/-/-/C. Wolf (2012f), Langfrist-Online-Tracking, T18, ZA5351, Studienbeschreibung, GESIS Datenarchiv, Köln

-/-/_/- (2013a), Langfrist-Medienagenda-Analyse, ZA5323, Studienbeschreibung, GESIS Datenarchiv, Köln

-/-/-/C. Wolf (2013b), Langfrist-Online-Tracking, T19, ZA5719, Studienbeschreibung, GESIS Datenarchiv, Köln

-/-/-/-/- (2013c), Landtagswahl Niedersachsen 2013, ZA5735, Studienbeschreibung, GESIS Datenarchiv, Köln

Revilla, M. (2010), Quality in Unimode and Mixed-Mode Designs: A Multitrait-Multimethod Approach, in: Survey Research Methods 4, 151-164

Rohrschneider, R./R. Schmitt-Beck/F. Jung (2012), Short-term Factors versus Longterm Values: Explaining the 2009 Election Results, in: Electoral Studies 31, 20-34

Roller, E. (1991), Ein analytisches Schema zur Klassifikation von Politikinhalten (FS III 91-201), Berlin, Wissenschaftszentrum Berlin für Sozialforschung

Römmele, A./S. Einwiller (2012), Soziale Medien in der Bundestagswahl 2009, in: Zeitschrift für Parlamentsfragen 1, 103-113

Roth, D. (2008), Empirische Wahlforschung. Ursprung, Theorien, Instrumente und Methoden, Wiesbaden

Rosenberg M. J./C. I. Hovland (1960): Cognitive, Affective and Behavioral Components of Attitudes, in: Hovland, C. I./M. J. Rosenberg (eds.), Attitude Organization and Change, New Haven, 1-14 
Roßteutscher, S. (2007), CDU-Wahl 2005: Katholiken, Kirchgänger und eine protestantische Spitzenkandidatin aus dem Osten, in: Brettschneider, F./O. Niedermayer/B. Weßels (Hg.), Die Bundestagswahl 2005. Analysen des Wahlkampfes und der Wahlergebnisse, Wiesbaden, 321-347

- (2012), Die konfessionell-religiöse Konfliktlinie zwischen Säkularisierung und Mobilisierung, in: PVS-Sonderheft 45 (Wählen in Deutschland), 111-133

Sanders, D./H. D. Clarke/M. C. Stewart/P. Whiteley (2007), Does Mode Matter for Modeling Political Choice? Evidence from the 2005 British Election Study, in: Political Analysis 15, 257-285

Scherer, P./I. E. Bieber (2013), Eintagsfliege oder Partei mit Zukunft? Eine empirische Untersuchung der Bevölkerungseinstellungen zur Piratenpartei, in: Mitteilungen des Instituts für Deutsches und Internationales Parteienrecht und Parteienforschung 19, 118-129

Scheuch, E. K. (2000), Die Kölner Wahlstudie zur Bundestagswahl 1961, in: Klein, M./W. Jagodzinski/E. Mochmann/D. Ohr (Hg.), 50 Jahre empirische Wahlforschung in Deutschland. Entwicklung, Befunde, Perspektiven, Daten, Wiesbaden, $41-58$

Schmitt-Beck, R. (2011), Spitzenkandidaten, in: Rattinger, H./S. Roßteutscher/R. Schmitt-Beck/B. Weßels et al. (2011), Zwischen Langeweile und Extremen: Die Bundestagswahl 2009, Baden-Baden, 205-221

- (2012a), Empirische Wahlforschung in Deutschland: Stand und Perspektiven zu Beginn des 21. Jahrhunderts, in: PVS-Sonderheft 45 (Wählen in Deutschland), 239

- (2012b), Campaign Media Content Analysis TV, ZA5306, Methodology Report, GESIS Datenarchiv, Köln

- (2012c), Campaign Media Content Analysis Print, ZA530\%, Methodology Report, GESIS Datenarchiv, Köln

- J. Partheymüller (2012), Why Voters Decide Late: A Simultaneous Test of Old and New Hypotheses at the 2005 and 2009 German Federal Elections, in: German Politics 21, 299-316

- H. Rattinger/S. Roßteutscher/B. Weßels (2010a), Die deutsche Wahlforschung und die German Longitudinal Election Study (GLES), in: Faulbaum, F./C. Wolf (Hg.), Gesellschaftliche Entwicklungen im Spiegel der empirischen Sozialforschung, Wiesbaden, 141-172

-/T. Faas/A. Wolsing (2010b), Kampagnendynamik bei der Bundestagswahl 2009. Die Rolling-Cross-Section-Studie im Rahmen der ,German Longitudinal Election Study ${ }^{6}$ 2009, Working Papers Nr. 134, Mannheimer Zentrum für Europäische Sozialforschung

-/J. Tenscher (2008), Divided We March, Divided We Fight: Trade Unions, Social Democrats, and Voters in the 2005 German General Election, in: Farrell, D. M./R. Schmitt-Beck (eds.), Non-Party-Actors in Electoral Politics. The Role of Interest Groups and Independent Citizens in Contemporary Election Campaigns, BadenBaden, 151-182

Schoen, H. (2005a), Wahlkampfforschung, in: Falter, J. W./H. Schoen (Hg.), Handbuch Wahlforschung, Wiesbaden, 503-542

- (2005b), Soziologische Ansätze in der empirischen Wahlforschung, in: Falter, J. W./H. Schoen (Hg.), Handbuch Wahlforschung, Wiesbaden, 135-185

- (2005c), Wechselwahl, in: Falter, J. W./H. Schoen (Hg.), Handbuch Wahlforschung, Wiesbaden, 367-387 
- (2009a), Wahlsoziologie, in: Kaina, V./A. Römmele (Hg.), Politische Soziologie. Ein Studienbuch, Wiesbaden, 181-208

- (2009b), Ossis und Wessis - Befunde zum Elektorat in Ost und West. Tagung zur Bundestagswahl 2009. Befunde, Bewertungen, Schlussfolgerungen, Nürnberg, 16.10.-18.10.2009

- (2010), Ein Bericht von der Heimatfront. Bürger, Politiker und der Afghanistaneinsatz der Bundeswehr, in: Politische Vierteljahresschrift 51, 395-408

-/C. Weins (2005), Der sozialpsychologische Ansatz zur Erklärung von Wahlverhalten, in: Falter, J. W./H. Schoen (Hg.), Handbuch Wahlforschung, Wiesbaden, 187242

Schroth, Y. (2003), Die Stichprobe, in: Wüst, A. (Hg.), Politbarometer, Opladen, 3750

Schultze, M. (2012), Effekte des Wahl-O-Mat auf politisches Wissen über Parteipositionen, in: Zeitschrift für Politikwissenschaft 22, 367-391

Steenbergen, M. R. (2010), The New Political Psychology of Voting, in: Faas, T./K. Arzheimer/S. Roßsteutscher (Hg.), Information - Wahrnehmung - Emotion. Politische Psychologie in der Wahl- und Einstellungsforschung, Wiesbaden, 13-31

Tenscher, J. (2007), Professionalisierung nach Wahl. Ein Vergleich der Parteikampagnen im Rahmen der jüngsten Bundestags- und Europawahlkämpfe in Deutschland, in: Brettschneider F./O. Niedermayer/B. Weßels (Hg.), Die Bundestagswahl 2005. Analysen des Wahlkampfes und der Wahlergebnisse, Wiesbaden, 65-95

- (2013), Ein Hauch von Wahlkampf, in: Weßels, B./H. Schoen/O. W. Gabriel (Hg.), Wahlen und Wähler. Analysen aus Anlass der Bundestagswahl 2009, Wiesbaden, 63-78

Thurner, P. W. (1998), Wählen als rationale Entscheidung. Die Modellierung von Politikreaktionen im Mehrparteiensystem, München

-/I. Mauerer/M. Binder (2012), Parteienspezifisches Issue-Voting bei den Bundestagswahlen 2002 bis 2009, in: PVS-Sonderheft 45 (Wählen in Deutschland), 302320

Wagner, A./B. Weßels (2012a), Kanzlerkandidaten - Wie beeinflussen sie die Wahlentscheidung?, in: PVS-Sonderheft 45 (Wählen in Deutschland), 345-370

- / - (2012b), Parties and Their Leaders. Does It Matter How They Match? The German General Elections 2009 in Comparison, in: Electoral Studies 31, 72-82

Weßels, B. (2011a), Schwankende Wähler: Gefährden Wechselwähler die Demokratie?, in: Bytzek, E./S. Roßteutscher (Hg.), Der unbekannte Wähler? Mythen und Fakten über das Wahlverhalten der Deutschen, Frankfurt a.M.-Wiesbaden, 43-57

- (2011b), Das Wahlverhalten sozialer Gruppen, in: Rattinger, H./S. Roßteutscher/R. Schmitt-Beck/B. Weßels et al. (Hg.), Zwischen Langeweile und Extremen: Die Bundestagswahl 2009, Baden-Baden, 103-117

Wüst, A. (2003), Politbarometer, Opladen

Zittel, T./T. Gschwend (2007), Der Bundestagswahlkampf von 2005 und Kollektive Repräsentation: Ein kurzer Auftakt zum langen Abschied?, in: Brettschneider, F./O. Niedermayer/B. Weßels (Hg.), Die Bundestagswahl 2005. Analysen des Wahlkampfes und der Wahlergebnisse, Wiesbaden, 119-144 